\title{
Convection in directionally solidifying alloys under inclined rotation
}

\author{
By C. A. CHUNG AND FALIN CHEN \\ Institute of Applied Mechanics, National Taiwan University, Taipei, Taiwan 10764, ROC
}

(Received 9 March 1999 and in revised form 3 September 1999)

In an experiment on binary alloys directionally solidifying from below, Sample \& Hellawell (1984) showed that the plume convection can be successfully prohibited by rotating the cooling tank around an inclined axis. In the present paper we interpret their experimental observation by an analytical approach. Results show that there is a flow induced by the inclination. The induced flow in the fluid layer is a parallel shear flow consisting of three parts: the thermal boundary-layer flow, the solute boundarylayer flow, and the Ekman-layer flow. In the mush, the induced flow is also a parallel flow but of much smaller velocity, consisting of two flows of opposite directions. The induced velocity in the fluid layer increases with inclination angle and decreases with the effective Taylor number $T_{e}$. The induced velocity in the mush also increases with inclination angle but remains virtually the same on varying the speed of rotation. The linear stability analysis of the mushy layer shows that, due mostly to the reduction of buoyancy, the mush becomes more stable as the inclination angle increases. In the precession-only case, the most-unstable mode of instability is the longitudinal mode, which propagates in a direction perpendicular to the induced flow. In the spin (with or without precession) case, the instability modes propagating in different directions are of equal stability. Because the induced flow changes direction with a frequency equal to the spin angular velocity, the flow scans over all the directions of the system and stabilizes equally the modes in different directions. We conclude on the basis of the present results and from the practical point of view that spin-only rotation is more effective than the precession-only rotation in stabilizing the convection during solidification.

\section{Introduction}

The directional solidification process has attracted much research effort for several decades due to its wide application in, for example, the semiconductor industry regarding single crystal growth and the aerospace industry regarding the manufacture of turbine blades for high-performance turbine engines. During the solidification process, a wide range of fluid-mechanical phenomena may occur. In particular, the convection due to buoyancy occurring adjacent to the freezing front (or the melt/solid interface) may profoundly influence the internal structure of the resultant castings (Langlois 1985; Davis 1990; Huppert 1990). For some alloys the melt/solid interface is morphologically unstable (Mullins \& Sekerka 1964), causing the interface to become highly convoluted and eventually to evolve into a dendritic mushy zone below the bulk melt and above the solid. As a result, several different kinds of buoyancy-driven convection occur in both the bulk melt and the dendritic mush. For details the reader 
may refer to the experiments conducted by, for example, Chen \& Chen (1991) and Tait \& Jaupart (1992).

In essence, at the onset of the convection in the solidifying system consisting of a fluid layer above and a mushy layer below, there are two different instability modes competing (Worster 1992; Chen, Lu \& Yang 1994). They are the boundarylayer mode, a convection confined to the shallow solute boundary layer above the melt/mush interface, and the mushy-layer mode, a convection circulating in the whole depth of the mush. For aqueous ammonium chloride solution, Chen et al. (1994) indicated that in a wide-range of physical parameters the boundary-layer mode dominates the system, and it will evolve into the nonlinear salt-finger convection as solidification proceeds. Under the strong perturbation imposed by the vigorous saltfinger convection above the interface, convection in the mush is eventually triggered subcritically (Amberg \& Homsy 1993; Anderson \& Worster 1995; Chung \& Chen 2000). Consequently, a large number of plumes containing buoyant, salt-depleted solution emanate from the interior of the mush. The plumes flow through essentially vertical channels, forming so-called 'chimneys' or the freckles in the casting. The freckles have a detrimental influence on the mechanical strength of the resultant castings, which is a major motivation for researchers to discover means to prevent their formation.

To date, there have been two experiments that have successfully shown a scheme to prevent the formation of chimneys. In the first experiment, by Sample \& Hellawell (1984), 25, 30 and $35 \mathrm{wt} \%$ ammonium chloride solutions were used. They rotated the cooling tank with respect to an axis of inclination angle $\phi_{n}$ varying from $0^{\circ}$ to $30^{\circ}$. Two cases of rotation were considered: the spin-only case and the spin-and-precession case. Both cases led to the same conclusion that, under the inclined rotation with $20^{\circ} \leqslant \phi_{n} \leqslant 30^{\circ}$, the plume convection can be effectively suppressed when the rotation speed is less than 5 r.p.m. For $\phi_{n} \leqslant 10^{\circ}$, the plumes were still observed. They did not investigate $\phi_{n}>30^{\circ}$ due to the limitation of the experimental facility. They attributed the success of the suppression of the plume to the shear flow occurring above the melt/mush interface, which inhibits the salt-finger convection in the solute boundary layer and in turn inhibits the occurrence of plumes. They pointed out further that rotation about a vertical axis could not effectively eliminate the plume, which has recently been confirmed by the analyses of Neilson \& Incropera (1993) and Lu \& Chen (1997).

The second experiment was carried out by Huppert \& Hallworth (1993), who added small quantities of copper sulphate to a solution of $27 \mathrm{wt} \%$ ammonium chloride in water. They found that as the quantity of the contaminant increased, the time taken for the plumes to appear in the system increased. When the contamination increased up to $0.5 \mathrm{wt} \%$, no plume was observed at all. They attributed this inhibition of the plume to the change of the morphology of the dendrites of the mush: as the contamination increased, the crystals became more faceted so that the solid fraction of the mush increased, leading to a higher resistance to the flow in the mush. Once the solid fraction was high enough, a complete suppression of the plumes was achieved. Later, Worster \& Kerr (1994) conducted an theoretical analysis to interpret the experimental observations of Huppert \& Hallworth.

In the present paper, we will focus on the first experiment and employ an analytical approach to interpret the experimental observations of Sample \& Hellawell (1984). We will first solve analytically the shear flow induced by the inclination of the system, which consists of a semi-infinite fluid layer above and a mushy layer below. It is found that the induced flow turns out to be a result of the interaction among gravity, the 
pressure gradient and the Coriolis force. Based on this induced flow, we implement a linear stability analysis to investigate the stability of the flow in the mush. The results can explain the experimental observations and shed light on the mechanisms stabilizing the system under inclined rotation. Note however that, instead of the spinonly and the spin-and-precession cases considered by Sample \& Hellawell, we will examine the precession-only and the spin-and-precession cases. This is because, as we shall point out later in the paper, as spin is imposed, its effect will predominate over the precession. This explains why the two cases considered by Sample \& Hellawell (both including spin) led to similar results.

In the following, the formulation of the system under a general rotating motion is given in $\S 2$. These equations and associated boundary conditions are solved analytically for the flow induced by the inclination in $\S 3$, in which several examples of the induced flow are given and the limits to the existence of solutions are discussed. The linearized perturbation equations for the linear stability analysis in the mush are derived in $\S 4$. Then the linear stability analyses for the precession-only case and the precession-and-spin case are shown in respectively $\S 5$ and $\S 6$. Finally, concluding remarks regarding the induced flow, the stability in the mush, and the limitation of the present solution are given in $\S 7$.

\section{Formulation and problem description}

Consider the system as shown in figure 1, a binary solution of concentration $C_{0}$ and temperature $T_{\infty}$ being unidirectionally solidified from below, in which a dendritic mush, extending from $z=0$ to $z=h(x, y, t)$, forms between the eutectic solid below and the semi-infinite bulk fluid above. The height of the mush $h$ is to be determined as part of the solution. Assume that the solid/mush interface moves in the direction of thermal gradient with a constant velocity $V$. The system rotates in a general way such that the angular velocity can be described as

$$
\dot{\boldsymbol{\Phi}}=\left(\dot{\phi}_{p} \sin \phi_{n} \sin \phi_{s}\right) \boldsymbol{e}_{x}+\left(\dot{\phi}_{p} \sin \phi_{n} \cos \phi_{s}\right) \boldsymbol{e}_{y}+\left(\dot{\phi}_{p} \cos \phi_{n}+\dot{\phi}_{s}\right) \boldsymbol{e}_{z},
$$

where $\phi_{p}, \phi_{n}$ and $\phi_{s}$ are the angles of precession, nutation and spin, respectively, and $\dot{\phi}_{p}$ and $\dot{\phi}_{s}$ are the angular velocities of precession and spin, respectively; $\boldsymbol{e}_{x}, \boldsymbol{e}_{y}$ and $\boldsymbol{e}_{z}$ are the unit vectors of a Cartesian coordinate system, which, after taking the Galilean transformation with respect to the interface velocity $V$, is fixed on the mush/solid interface. In figure 1 the coordinate system is denoted by $x, y, z$ with heavy lines.

Two set of equations, one in the fluid layer (or the bulk melt) and one in the mushy layer, are required to describe the fluid motion of the system (or the cooling tank). The coordinate system is fixed on the mush/solid interface, translating with $V$ and rotating with $\dot{\boldsymbol{\Phi}}$, and the governing equations in the fluid layer $h<z<\infty$ are

$$
\nabla \cdot u=0
$$

$$
\begin{aligned}
{\left[\frac{\partial}{\partial t}-V \frac{\partial}{\partial z}+\boldsymbol{u} \cdot \nabla\right] \boldsymbol{u} } & =-\nabla P+v \nabla^{2} \boldsymbol{u}+\left(\frac{\rho}{\rho_{0}}-1\right) \boldsymbol{g}+2 \boldsymbol{u} \times \dot{\boldsymbol{\Phi}} \\
\left(\frac{\partial}{\partial t}-V \frac{\partial}{\partial z}+\boldsymbol{u} \cdot \nabla\right) T & =\kappa \nabla^{2} T \\
\left(\frac{\partial}{\partial t}-V \frac{\partial}{\partial z}+\boldsymbol{u} \cdot \nabla\right) C & =D_{f} \nabla^{2} C
\end{aligned}
$$



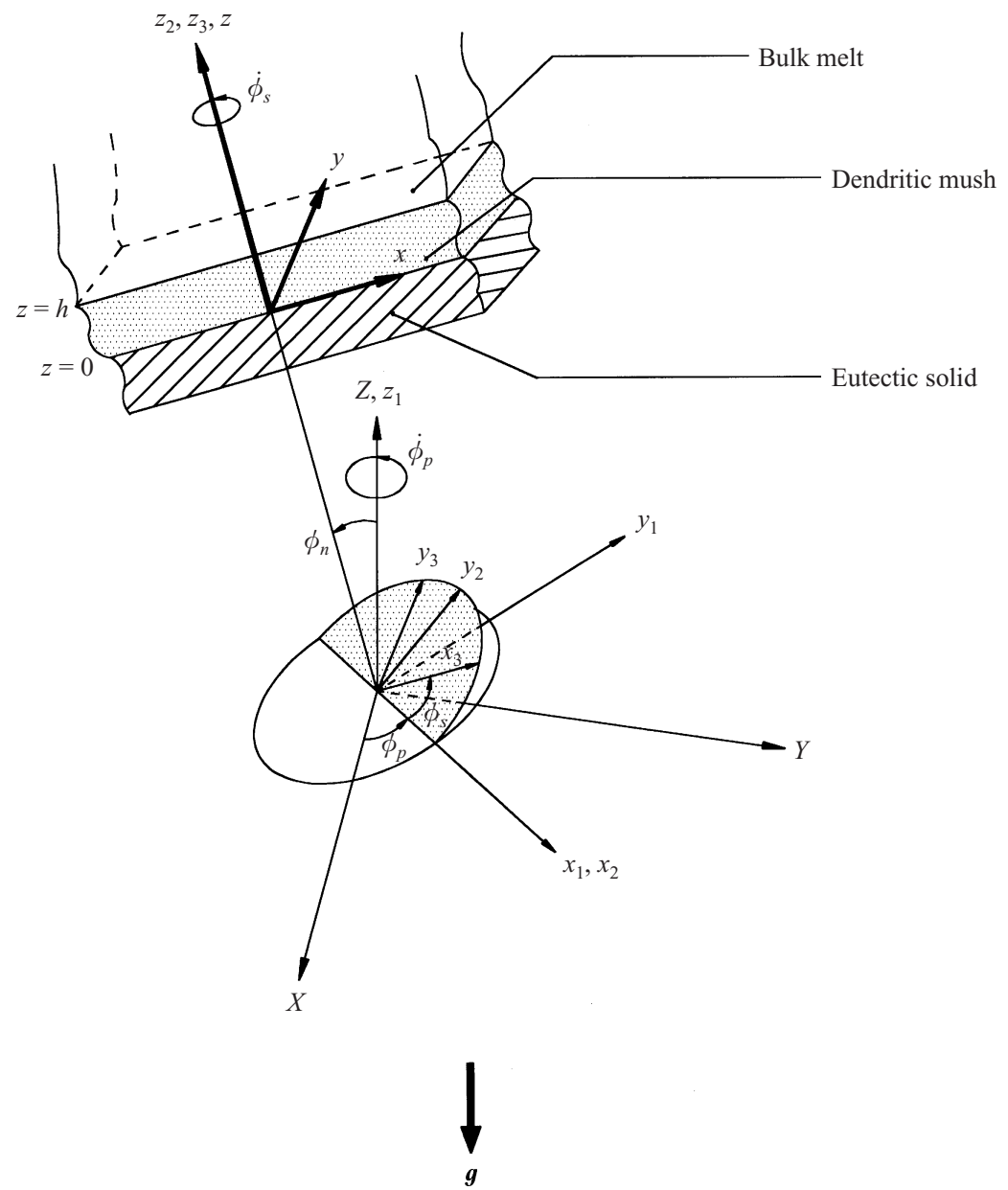

Figure 1. The system considered. A semi-infinite fluid layer (or the bulk melt) overlies a dendritic mushy layer. The $x, y, z$ coordinate system is fixed on the mush/solid interface. The inertia frame is denoted by $X, Y, Z$, which after several coordinate transformations shown in the figure becomes the $x, y, z$ system. $\phi_{n}$ denotes the inclination angle, $\phi_{p}$ denotes the precession angle, and $\phi_{s}$ denotes the spin angle. Gravity points vertically downwards.

In the above equations, $\boldsymbol{u}$ is the velocity vector $(u, v, w)$ with respect to the cooling tank, $P=p / \rho_{0}-\boldsymbol{g} \cdot \boldsymbol{r}, p$ is the static pressure, $\rho_{0}$ the reference density, $\boldsymbol{r}$ the position vector, $T$ the temperature, $C$ the concentration, $\kappa$ the thermal diffusivity, $D_{f}$ the solute diffusivity, $v$ the kinematic viscosity, and $\boldsymbol{g}=-g\left(\sin \phi_{n} \sin \phi_{s}, \sin \phi_{n} \cos \phi_{s}, \cos \phi_{n}\right)$ the gravitational acceleration vector, depending on both the nutation and spin angles, and $g$ is the gravitational constant.

In the momentum equation $(2.2 b)$, we have neglected the centrifugal accelaration $\left(\rho / \rho_{0}\right) \dot{\boldsymbol{\Phi}} \times(\dot{\boldsymbol{\Phi}} \times \boldsymbol{r})$ as well as the tangential acceleration $\left(\rho / \rho_{0}\right) \ddot{\boldsymbol{\Phi}} \times \boldsymbol{r}$. The relative importance of these two terms compared to the gravity force is determined by the ratio $|\dot{\boldsymbol{\Phi}}|^{2} l_{c} / g$, where $l_{c}$ is the characteristic length measured from the rotation axis. Since the rotation speed considered in the present paper is about $1-10$ r.p.m. and $l_{c}$ is about $20 \mathrm{~cm}$ (the dimension of the cooling tank, Sample \& Hellawell 1984), the ratio is less than 0.02 . Therefore, both the centrifugal and tangential terms can be 
neglected in the present analysis. Since the Boussinesq approximation is applied, the density of the fluid is a constant except in the gravity term in which the following relation holds:

$$
\rho=\rho_{0}\left(1-\alpha^{*}\left(T-T_{L}\left(C_{0}\right)\right)+\beta^{*}\left(C-C_{0}\right)\right),
$$

where $\alpha^{*}$ and $\beta^{*}$ are respectively thermal and solute expansion coefficients, and $T_{L}\left(C_{0}\right)$ is the liquidus temperature corresponding to $C_{0}$.

In the mushy layer $0<z<h$, the governing equations are

$$
\begin{gathered}
\nabla \cdot \boldsymbol{u}=0, \\
\frac{v}{\Pi(\chi)} \boldsymbol{u}=-\nabla P+\left(\frac{\rho}{\rho_{0}}-1\right) \boldsymbol{g}+\frac{2}{\chi} \boldsymbol{u} \times \dot{\boldsymbol{\Phi}}, \\
\left(\frac{\partial}{\partial t}-V \frac{\partial}{\partial z}+\boldsymbol{u} \cdot \nabla\right) T=\kappa \nabla^{2} T-\frac{\mathscr{L}}{c}\left(\frac{\partial}{\partial t}-V \frac{\partial}{\partial z}\right) \chi, \\
\left(\frac{\partial}{\partial t}-V \frac{\partial}{\partial z}\right)\left[\chi C+(1-\chi) C^{*}\right]+\boldsymbol{u} \cdot \nabla C=\nabla \cdot\left(\chi D_{f} \nabla C\right) .
\end{gathered}
$$

In (2.4), $\Pi(\chi)$ is the permeability function, $\chi$ the porosity, $\mathscr{L}$ the latent heat per unit volume, $c$ the specific heat per unit volume, and $C^{*}$ the concentration of the solid. The specific heat coefficients, the thermal conductivities and the solute diffusivities of both the liquid and solid phases are assumed to be equal. Moreover, as discussed for $(2.2 b)$, the centrifugal acceleration and tangential acceleration due to the rotation have also been neglected in the Darcy equation $(2.4 b)$. Since the thermodynamic equilibrium condition holds in the mush (Chen et al. 1994), the liquidus relation

$$
T-T_{L}\left(C_{0}\right)=\Gamma\left(C-C_{0}\right)
$$

is applied, where $\Gamma$ is the slope of the liquidus. As a result, the density can be represented by

$$
\rho=\rho_{0}\left(1+\bar{\beta}\left(C-C_{0}\right)\right),
$$

where $\bar{\beta}=\beta^{*}-\Gamma \alpha^{*}$. Note that the Coriolis acceleration $2 \boldsymbol{u} \times \dot{\boldsymbol{\Phi}}$ in both $(2.2 b)$ and (2.4b) can be expressed as

$$
\begin{aligned}
2 \boldsymbol{u} \times \dot{\boldsymbol{\Phi}}=2\left[\left(\dot{\phi}_{p} C_{n}+\dot{\phi}_{s}\right) v-\right. & \left.\left(\dot{\phi}_{p} S_{n} C_{s}\right) w\right] \boldsymbol{e}_{x}+2\left[-\left(\dot{\phi}_{p} C_{n}+\dot{\phi}_{s}\right) u\right. \\
& \left.+\left(\dot{\phi}_{p} S_{n} S_{s}\right) w\right] \boldsymbol{e}_{y}+2\left[\left(\dot{\phi}_{p} S_{n} C_{s}\right) u-\left(\dot{\phi}_{p} S_{n} S_{s}\right) v\right] \boldsymbol{e}_{z} .
\end{aligned}
$$

In (2.7), we have used the abbreviations

$$
S_{n} \equiv \sin \phi_{n}, \quad C_{n} \equiv \cos \phi_{n}, \quad S_{s} \equiv \sin \phi_{s}, \quad C_{s} \equiv \cos \phi_{s},
$$

which will be also used in the subsequent formulations.

The boundary conditions at the infinite far field are that both the temperature and concentration are assumed to remain the same as the original solution and the fluid experiences a rigid-body rotation. Also, the height of the tank is assumed large enough that the influence due to the possible deformation of the free surface can be neglected. Accordingly, as $z \rightarrow \infty$ we have

$$
T \rightarrow T_{\infty}, \quad C \rightarrow C_{0}, \quad \boldsymbol{u} \rightarrow \mathbf{0} .
$$


At the melt/mush interface $z=h(x, y, t)$ the boundary conditions are

$$
\begin{gathered}
T-T_{L}\left(C_{0}\right)=\Gamma\left(C-C_{0}\right), \quad \frac{\partial T}{\partial n}=\Gamma \frac{\partial C}{\partial n}, \quad[\boldsymbol{u} \cdot \boldsymbol{n}]=0, \\
{[T]=0, \quad\left[\frac{\partial T}{\partial n}\right]=0, \quad \chi=1, \quad\left[\sigma_{n}\right]=0,} \\
\left.\frac{\partial \boldsymbol{u}_{2}}{\partial n}\right|_{h^{+}}=\frac{\Lambda}{\sqrt{\Pi(1)}}\left(\left.\boldsymbol{u}_{2}\right|_{h^{+}}-\left.\boldsymbol{u}_{2}\right|_{h^{-}}\right),
\end{gathered}
$$

where [ ] denotes the jump of the enclosed quantity across the interface, $\boldsymbol{n}$ is the normal vector to the interface, and $\sigma_{n}$ is the normal stress. Equation (2.10a) represents concentration continuity, $(2.10 \mathrm{~b})$ is the marginal equilibrium condition proposed by Worster (1986), $(2.10 c)$ and $(2.10 d)$ account respectively for mass and temperature continuity, $(2.10 e)$ represents heat flux continuity, $(2.10 \mathrm{f})$ means that the porosity is fixed to unity, $(2.10 \mathrm{~g})$ denotes normal stress continuity, and $(2.10 \mathrm{~h})$ is an empirical condition proposed by Beavers \& Joseph (1967), describing the relation between the planar velocities in the fluid and mushy layers, in which $\boldsymbol{u}_{2}$ is the planar velocity vector $(u, v)$ and $\Lambda$ is an empirical constant determined by experiment. The boundary conditions at mush/solid interface are that the eutectic concentration (or temperature) and impermeable boundary are prescribed; namely, at $z=0$ we have

$$
T=T_{E}, \quad \boldsymbol{u} \cdot \boldsymbol{n}=0,
$$

where $T_{E}$ is the eutectic temperature of the solid.

We non-dimensionalize the governing equations as well as the boundary conditions with the following scales: $V$ for velocity, $H=\kappa / V$ for length, $\kappa / V^{2}$ for time, and $\bar{\beta} \Delta C \rho_{0} g \kappa / V$ for pressure in which, $\Delta C=C_{0}-C_{E}$ and $C_{E}$ is the eutectic concentration. The dimensionless temperature and concentration are respectively

$$
\theta=\frac{T-T_{L}\left(C_{0}\right)}{\Delta T}, \quad \Theta=\frac{C-C_{0}}{\Delta C},
$$

where $\Delta T=\Gamma \Delta C=T_{L}\left(C_{0}\right)-T_{E}$. For convenience we adopt the same notation as in the previous equations for the dimensionless quantities in the following equations. We consider in the present paper a particular rotation in which both $\dot{\phi}_{p}$ and $\dot{\phi}_{s}$ are fixed to be constant and $\dot{\phi}_{n}=0$, i.e. the nutation motion is absent from the present system and the nutation angle $\phi_{n}$, or the inclination angle, is fixed. As a result, in the fluid layer, the dimensionless continuity equation, momentum equation, heat balance and solute balance equations are respectively

$$
\begin{aligned}
\nabla \cdot \boldsymbol{u}=0 & \\
\frac{1}{\sigma}\left[\frac{\partial}{\partial t}-\frac{\partial}{\partial z}+\boldsymbol{u} \cdot \nabla\right] u= & \nabla^{2} u-\mathscr{H} R_{m} \frac{\partial p}{\partial x}+\left(R_{t} \theta-R_{c} \Theta\right) S_{n} S_{s} \\
& +\left[(-1)^{n_{p}} T_{a p}^{1 / 2} C_{n}+(-1)^{n_{s}} T_{a s}^{1 / 2}\right] v-\left[(-1)^{n_{p}} T_{a p}^{1 / 2} S_{n} C_{s}\right] w, \\
\frac{1}{\sigma}\left[\frac{\partial}{\partial t}-\frac{\partial}{\partial z}+\boldsymbol{u} \cdot \nabla\right] v= & \nabla^{2} v-\mathscr{H} R_{m} \frac{\partial p}{\partial y}+\left(R_{t} \theta-R_{c} \Theta\right) S_{n} C_{s} \\
& -\left[(-1)^{n_{p}} T_{a p}^{1 / 2} C_{n}+(-1)^{n_{s}} T_{a s}^{1 / 2}\right] u+\left[(-1)^{n_{p}} T_{a p}^{1 / 2} S_{n} S_{s}\right] w,
\end{aligned}
$$




$$
\begin{aligned}
\frac{1}{\sigma}\left[\frac{\partial}{\partial t}-\frac{\partial}{\partial z}+\boldsymbol{u} \cdot \nabla\right] w= & \nabla^{2} w-\mathscr{H} R_{m} \frac{\partial p}{\partial z}+\left(R_{t} \theta-R_{c} \Theta\right) C_{n} \\
& +\left[(-1)^{n_{p}} T_{a p}^{1 / 2} S_{n} C_{s}\right] u-\left[(-1)^{n_{p}} T_{a p}^{1 / 2} S_{n} S_{s}\right] v \\
& \left(\frac{\partial}{\partial t}-\frac{\partial}{\partial z}+\boldsymbol{u} \cdot \nabla\right) \theta=\nabla^{2} \theta \\
& \left(\frac{\partial}{\partial t}-\frac{\partial}{\partial z}+\boldsymbol{u} \cdot \nabla\right) \Theta=\epsilon \nabla^{2} \Theta
\end{aligned}
$$

In the mushy layer, the continuity equation, Darcy equation, heat and solute balance equations are respectively

$$
\nabla \cdot \boldsymbol{u}=0
$$

$$
\frac{u}{\Pi(\chi)}=-R_{m} \frac{\partial p}{\partial x}-R_{m} S_{n} S_{s} \theta+\left[(-1)^{n_{p}} T_{m p}^{1 / 2} C_{n}+(-1)^{n_{s}} T_{m s}^{1 / 2}\right] \frac{v}{\chi}-\left[(-1)^{n_{p}} T_{m p}^{1 / 2} S_{n} C_{s}\right] \frac{w}{\chi},
$$

$$
\frac{v}{\Pi(\chi)}=-R_{m} \frac{\partial p}{\partial y}-R_{m} S_{n} C_{s} \theta-\left[(-1)^{n_{p}} T_{m p}^{1 / 2} C_{n}+(-1)^{n_{s}} T_{m s}^{1 / 2}\right] \frac{u}{\chi}+\left[(-1)^{n_{p}} T_{m p}^{1 / 2} S_{n} S_{s}\right] \frac{w}{\chi}
$$

$$
\begin{gathered}
\frac{w}{\Pi(\chi)}=-R_{m} \frac{\partial p}{\partial z}-R_{m} C_{n} \theta+\left[(-1)^{n_{p}} T_{m p}^{1 / 2} S_{n} C_{s}\right] \frac{u}{\chi}-\left[(-1)^{n_{p}} T_{m p}^{1 / 2} S_{n} S_{s}\right] \frac{v}{\chi} \\
\left(\frac{\partial}{\partial t}-\frac{\partial}{\partial z}+\boldsymbol{u} \cdot \nabla\right) \theta=\nabla^{2} \theta-\mathscr{F}\left(\frac{\partial}{\partial t}-\frac{\partial}{\partial z}\right) \chi \\
\chi\left(\frac{\partial}{\partial t}-\frac{\partial}{\partial z}\right) \theta+\boldsymbol{u} \cdot \nabla \theta=-(\theta-\mathscr{C})\left(\frac{\partial}{\partial t}-\frac{\partial}{\partial z}\right) \chi .
\end{gathered}
$$

In the above equations, $\sigma \equiv v / \kappa$ is the Prandtl number, $\mathscr{H} \equiv H^{2} / \Pi_{0}$ is the inverse Darcy number, $\Pi_{0}$ is the reference permeability of the mush, $R_{t}$ and $R_{c}$ are respectively the thermal and solute Rayleigh numbers of the fluid layer, and $R_{m}$ is the Rayleigh number of the mushy layer. They are defined as

$$
R_{t} \equiv \frac{g \alpha^{*} \Delta T H^{3}}{\kappa v}, \quad R_{c} \equiv \frac{g \beta^{*} \Delta C H^{3}}{\kappa v}, \quad R_{m} \equiv \frac{g \bar{\beta} \Delta C \Pi_{0} H}{\kappa v} .
$$

$R_{t}, R_{c}$ and $R_{m}$ satisfy the relations

$$
R_{t}=\mathscr{A} \mathscr{H} R_{m}, \quad R_{c}=(1+\mathscr{A}) \mathscr{H} R_{m},
$$

where $\mathscr{A} \equiv \Gamma \alpha^{*} / \bar{\beta}$ is the buoyancy ratio. In addition, $\varepsilon \equiv D_{f} / \kappa$ is the inverse Lewis number, $\mathscr{F} \equiv \mathscr{L} /(c \Delta T)$ is the Stephan number, and $\mathscr{C} \equiv\left(C^{*}-C_{0}\right) / \Delta C$ is the concentration ratio. Since $\varepsilon$ in the mush is very small, we neglect the mass diffusion in the mush. Also, because of the thermodynamic equilibrium condition, (2.5), the non-dimensional liquidus relation in the mush can be written as $\theta=\Theta$, which has been introduced into $(2.14 b, c, d, f)$. Note that since the nutation angle $\phi_{n}$ is considered fixed, $S_{n}$ and $C_{n}$ in $(2.8 a, b)$ are now constants. Nevertheless, because of the spin, the 
spin angle is a function of time, so that $(2.8 c, d)$ can be rewritten as

$$
S_{s}(t) \equiv \sin \phi_{s}=\sin (\Omega t), \quad C_{s}(t) \equiv \cos \phi_{s}=\cos (\Omega t),
$$

namely both $S_{s}$ and $C_{s}$ are periodical functions of time with frequency $\Omega=$ $\frac{1}{2} \sigma(-1)^{n_{s}} T_{a s}^{1 / 2}$, which is equivalent to the non-dimensional $\dot{\phi}_{s}$. The dimensionless parameters $T_{a p}$ and $T_{a s}$ are respectively the Taylor numbers for precession and spin, defined as

$$
T_{a p} \equiv\left(\frac{2 H^{2} \dot{\phi}_{p}}{v}\right)^{2}, \quad T_{a s} \equiv\left(\frac{2 H^{2} \dot{\phi}_{s}}{v}\right)^{2},
$$

and, similarly, $T_{m p}$ and $T_{m s}$ are respectively the Taylor numbers for precession and spin of the mush, defined as

$$
T_{m p} \equiv\left(\frac{2 \Pi_{0} \dot{\phi}_{p}}{v}\right)^{2}=\frac{T_{a p}}{\mathscr{H}^{2}}, \quad T_{m s} \equiv\left(\frac{2 \Pi_{0} \dot{\phi}_{s}}{v}\right)^{2}=\frac{T_{a s}}{\mathscr{H}^{2}} .
$$

We note that the exponents $n_{p}$ and $n_{s}$ account respectively for the sense of precession and spin. For $\dot{\phi}_{p}>0$ or $\dot{\phi}_{s}>0$, respectively $n_{p}=0$ or $n_{s}=0$, and for $\dot{\phi}_{p}<0$ or $\dot{\phi}_{s}<0$, respectively $n_{p}=1$ or $n_{s}=1$.

The boundary conditions as $z \rightarrow \infty$ are

$$
\theta \rightarrow \theta_{\infty}, \quad \Theta \rightarrow 0, \quad \boldsymbol{u} \rightarrow \mathbf{0},
$$

at the melt/mush interface $z=h(x, y, t)$ they are

$$
\begin{gathered}
\theta=\Theta, \quad \frac{\partial \theta}{\partial n}=\frac{\partial \Theta}{\partial n}, \quad[\boldsymbol{u} \cdot \boldsymbol{n}]=0, \quad[\theta]=0, \\
{\left[\frac{\partial \theta}{\partial n}\right]=0, \quad \chi=1, \quad\left[\sigma_{n}\right]=0,} \\
\left.\frac{\partial \boldsymbol{u}_{2}}{\partial n}\right|_{h^{+}}=\Lambda \sqrt{\frac{\mathscr{H}}{\Pi(1)}}\left(\left.\boldsymbol{u}_{2}\right|_{h^{+}}-\left.\boldsymbol{u}_{2}\right|_{h^{-}}\right),
\end{gathered}
$$

and at $z=0$ they are

$$
\theta=-1, \quad \boldsymbol{u} \cdot \boldsymbol{n}=0 .
$$

In the following, based on (2.13) and (2.14) and the associated boundary conditions (2.20-2.22), the flow induced by inclination and modified by rotation will be derived analytically and the stability based on the induced flow will be analysed through a numerical approach.

\section{Flow induced by inclination}

Before the onset of convection due to solidification, there is a flow induced by the inclination of the system (Sample \& Hellawell 1984; Neilson \& Incropera 1993). The induced flow is a result of the interaction between gravity and the pressure gradient and modified by the Coriolis force, which will be investigated in this section. Two kinds of rotation are considered: precession only and precession and spin. The solutions for the induced flow are obtained under some assumptions, which will also be discussed so that the requirements for the existence of the solutions can be clarified. 


\subsection{Analytical solution}

To investigate the induced flow, we need to solve (2.13) and (2.14) and the associated boundary conditions (2.20-2.22), which are however too complicated to be solved without assumptions. To simplify the problem, we assume that the horizontal dimension of the system (or the cooling tank) $L$ is much larger than the characteristic height of solidification $H$ (which is about $1 \mathrm{~cm}$ in the experiment of Sample \& Hellawell 1984), i.e.

$$
\frac{L}{H} \gg 1
$$

When small-scale convection is absent from the system, a scale analysis of the continuity equation yields

$$
\frac{U}{L} \approx \frac{W}{H},
$$

where $U$ and $W$ are respectively the characteristic velocities in the horizontal and vertical directions. From (3.1) and (3.2) we obtain $W \ll U$. Meanwhile, to satisfy (3.1), one can also derive from the scale analysis that the diffusion in the horizontal direction is negligible when compared to that in the vertical direction, i.e.

$$
\frac{\partial^{2}}{\partial x^{2}} \ll \frac{\partial^{2}}{\partial z^{2}}, \quad \frac{\partial^{2}}{\partial y^{2}} \ll \frac{\partial^{2}}{\partial z^{2}} .
$$

Due to the absence of small-scale fluid motion, the nonlinear advection terms, such as the third term on the left-hand side of $(2.13 b-f)$ and $(2.14 e, f)$, are much smaller than the pulling advection terms due to solidification, such as the second term (containing $\partial / \partial z$ ) on the left-hand side of these equations. This requires equivalently that $U / L \ll V / H$ or, after rewriting

$$
\frac{L}{H} \gg \frac{U}{V}
$$

Consequently, the temperature and concentration can be decoupled from the velocity. In other words, the temperature and concentration are not influenced by the presence of the flow induced by the inclination, and therefore will be the same as those in, for example, Worster (1992) or Chen et al. (1994), in which the system is motionless before the onset of convection. For convenience for the subsequent discussion, both the temperature and the concentration are shown in the following. In the fluid layer, they are

$$
\theta_{b}=\theta_{\infty}+\left(\theta_{i}-\theta_{\infty}\right) \exp \left[-\left(z-h_{b}\right)\right], \quad \Theta_{b}=\theta_{i} \exp \left[-\left(\frac{z-h_{b}}{\varepsilon}\right)\right],
$$

where $\theta_{i}$ is the temperature (or concentration) at the melt/mush interface

$$
\theta_{i}=\left(\frac{-\varepsilon}{1-\varepsilon}\right) \theta_{\infty}
$$

and $h_{b}$ is the mushy-layer height, which is a constant. In the mushy layer the temperature distribution $\theta_{b}$ is expressed by the implicit function

$$
z=\frac{\alpha-\mathscr{C}}{\alpha-\beta} \ln \left(\frac{\alpha+1}{\alpha-\theta_{b}}\right)+\frac{\mathscr{C}-\beta}{\alpha-\beta} \ln \left(\frac{\beta+1}{\beta-\theta_{b}}\right),
$$


and the porosity is a function of $\theta_{b}$

$$
\chi_{b}=\frac{\mathscr{C}-\theta_{i}}{\mathscr{C}-\theta_{b}}
$$

where

$$
\alpha=A+B, \quad \beta=A-B, \quad A=\frac{1}{2}\left(\mathscr{C}+\theta_{\infty}+\mathscr{F}\right), \quad B=\sqrt{A^{2}-\mathscr{C} \theta_{\infty}-\mathscr{F} \theta_{i}} .
$$

In the above equations the subscript $b$ denotes the basic state. The mushy-layer height $h_{b}$ can be obtained by substituting $\theta_{b}=\theta_{i}$ into (3.7).

With regard to the induced flow, taking into account $(2.13 b-d)$ and applying the rigid-body rotation condition $(2.20 \mathrm{c})$, we find that the pressure distribution can be expressed as

$$
p_{b}=\bar{p}_{b}(z, t)+\mathscr{A} \theta_{\infty}\left[x S_{n} S_{s}(t)+y S_{n} C_{s}(t)+z C_{n}\right] .
$$

We substitute (3.5)-(3.7) and (3.10) into (2.13b-d) and (2.14b-d) and find that the velocity and the pressure satisfy the following equations. In the fluid layer they are

$$
\begin{gathered}
{\left[\frac{1}{\sigma}\left(\frac{\partial}{\partial t}-\frac{\partial}{\partial z}\right)-\frac{\partial^{2}}{\partial z^{2}}\right] u_{b}-\left[(-1)^{n_{p}} T_{a p}^{1 / 2} C_{n}+(-1)^{n_{s}} T_{a s}^{1 / 2}\right] v_{b}} \\
=\left[R_{t}\left(\theta_{b}-\theta_{\infty}\right)-R_{c} \Theta_{b}\right] S_{n} S_{s}(t), \\
{\left[\frac{1}{\sigma}\left(\frac{\partial}{\partial t}-\frac{\partial}{\partial z}\right)-\frac{\partial^{2}}{\partial z^{2}}\right] v_{b}+\left[(-1)^{n_{p}} T_{a p}^{1 / 2} C_{n}+(-1)^{n_{s}} T_{a s}^{1 / 2}\right] u_{b}} \\
=\left[R_{t}\left(\theta_{b}-\theta_{\infty}\right)-R_{c} \Theta_{b}\right] S_{n} C_{s}(t), \\
w_{b}=0, \\
\mathscr{H} R_{m} \frac{\partial \bar{p}_{b}}{\partial z}=\left[(-1)^{n_{p}} T_{a p}^{1 / 2} S_{n} C_{s}(t)\right] u_{b}-\left[(-1)^{n_{p}} T_{a p}^{1 / 2} S_{n} S_{s}(t)\right] v_{b}+\left[R_{t}\left(\theta_{b}-\theta_{\infty}\right)-R_{c} \Theta_{b}\right] C_{n},
\end{gathered}
$$

and in the mushy layer they are

$$
\begin{gathered}
\frac{u_{b}}{\Pi_{b}}-\left[(-1)^{n_{p}} T_{m p}^{1 / 2} C_{n}+(-1)^{n_{s}} T_{m s}^{1 / 2}\right] \frac{v_{b}}{\chi_{b}}=-R_{m}\left(\mathscr{A} \theta_{\infty}+\theta_{b}\right) S_{n} S_{s}(t), \\
\frac{v_{b}}{\Pi_{b}}+\left[(-1)^{n_{p}} T_{m p}^{1 / 2} C_{n}+(-1)^{n_{s}} T_{m s}^{1 / 2}\right] \frac{u_{b}}{\chi_{b}}=-R_{m}\left(\mathscr{A} \theta_{\infty}+\theta_{b}\right) S_{n} C_{s}(t), \\
w_{b}=0, \\
R_{m} \frac{\partial \bar{p}_{b}}{\partial z}=\left[(-1)^{n_{p}} T_{m p}^{1 / 2} S_{n} C_{s}(t)\right] \frac{u_{b}}{\chi_{b}}-\left[(-1)^{n_{p}} T_{m p}^{1 / 2} S_{n} S_{s}(t)\right] \frac{v_{b}}{\chi_{b}}-R_{m}\left(\mathscr{A} \theta_{\infty}+\theta_{b}\right) C_{n} .
\end{gathered}
$$

The associated boundary conditions as $z \rightarrow \infty$ are

$$
u_{b} \rightarrow 0, \quad v_{b} \rightarrow 0
$$

and at $z=h_{b}$ they are

$$
\frac{\partial u_{b_{+}}}{\partial z}=\Lambda \sqrt{\frac{\mathscr{H}}{\Pi(1)}}\left(u_{b+}-u_{b-}\right), \quad \frac{\partial v_{b+}}{\partial z}=\Lambda \sqrt{\frac{\mathscr{H}}{\Pi(1)}}\left(v_{b+}-v_{b-}\right), \quad \bar{p}_{b+}=\bar{p}_{b-} .
$$


The pressure distribution (3.10) plays an interesting and important role in the basic state. The $z$-component of the pressure gradient balances the $z$-component of the fluid mass in both the liquid and mushy layers, leading to $w_{b}=0$ in both layers. In the far field, the $x$ - and $y$-components of the pressure gradient also balance the corresponding components of the mass of the fluid. Consequently, the fluid is motionless in the far field while it moves along the interface due to the imbalance between the fluid mass and the pressure gradient. Namely, near the melt/mush interface the buoyancy fails to balance the fluid mass in the $(x, y)$-plane when the tank is tilted.

Since in the mushy layer $(3.12 a, b)$ are algebraic equations, they can be easily solved and the solution after rearrangement is

$$
\begin{aligned}
u_{b} & \equiv u_{b}+\mathrm{i} v_{b} \\
& =\frac{S_{n} R_{m}\left(\mathscr{A} \theta_{\infty}+\theta_{b}\right)}{\Delta_{m}(z)} \exp \left[\mathrm{i}\left(\phi_{g}-\phi_{m}(z)\right)\right],
\end{aligned}
$$

where

$$
\phi_{g}=-\left(\Omega t+\frac{1}{2} \pi\right),
$$

denoting the orientation of the gravity component in the $(x, y)$-plane, which changes with time due to spin,

$$
\Delta_{m}(z)=\left[\frac{1}{\Pi_{b}^{2}}+\left(\frac{(-1)^{n_{p}} T_{m p}^{1 / 2} C_{n}+(-1)^{n_{s}} T_{m s}^{1 / 2}}{\chi_{b}}\right)^{2}\right]^{1 / 2},
$$

and the phase angle $\phi_{m}$ satisfies the relations

$$
\cos \phi_{m}=\frac{1}{\Pi_{b} \Delta_{m}}, \quad \sin \phi_{m}=\frac{(-1)^{n_{p}} T_{m p}^{1 / 2} C_{n}+(-1)^{n_{s}} T_{m s}^{1 / 2}}{\chi_{b} \Delta_{m}},
$$

The velocity is a periodic function, changing with time with a frequency equal to the spin angular velocity $\Omega$.

To solve the velocity in the fluid layer, we let

$$
U_{b} \equiv u_{b}+\mathrm{i} v_{b}=\hat{U}_{b} \exp \left(\mathrm{i} \phi_{g}\right) .
$$

Substituting this into $(3.11 a, b)$ yields an ordinary differential equation. We solve this equation by a formal approach and obtain the solution

$$
\begin{aligned}
\hat{U}_{b}= & \frac{S_{n} R_{t}\left(\theta_{i}-\theta_{\infty}\right)}{\Delta_{t}} \exp \left[\mathrm{i} \phi_{t}-\left(z-h_{b}\right)\right]-\frac{S_{n} R_{c} \theta_{i}}{\Delta_{c}} \exp \left[\mathrm{i} \phi_{c}-\left(z-h_{b}\right) / \varepsilon\right] \\
& +\left\{-\left(1+\Lambda \sqrt{\frac{\mathscr{H}}{\Pi(1)}}\right) \frac{S_{n} R_{t}\left(\theta_{i}-\theta_{\infty}\right)}{\Delta_{i} \Delta_{c}} \exp \left(\mathrm{i}\left[\phi_{t}-\phi_{i}-b\left(z-h_{b}\right)\right]\right)\right. \\
& +\left(\frac{1}{\varepsilon}+\Lambda \sqrt{\frac{\mathscr{H}}{\Pi(1)}}\right) \frac{S_{n} R_{c} \theta_{i}}{\Delta_{i} \Delta_{c}} \exp \left(\mathrm{i}\left[\phi_{c}-\phi_{i}-b\left(z-h_{p}\right)\right]\right) \\
& \left.+\Lambda \sqrt{\frac{\mathscr{H}}{\Pi(1)}} \frac{S_{n} R_{m}\left(\mathscr{A} \theta_{\infty}+\theta_{i}\right)}{\Delta_{i} \Delta_{m}\left(h_{b}\right)} \exp \left(-\mathrm{i}\left[\phi_{m}\left(h_{b}\right)+\phi_{i}+b\left(z-h_{b}\right)\right]\right)\right\} \\
& \times \exp \left[-\left(z-h_{b}\right) / d_{E}\right],
\end{aligned}
$$


in which

$$
\begin{gathered}
d_{E}=\frac{1}{((1 / 2 \sigma)+a)}, \quad a=\left[\frac{1}{2}\left(\frac{1}{4 \sigma^{2}}+\sqrt{\frac{1}{16 \sigma^{4}}+T_{e}^{2}}\right)\right]^{1 / 2}, \\
b=\frac{T_{e}}{\left|T_{e}\right|}\left[\frac{1}{2}\left(-\frac{1}{4 \sigma^{2}}+\sqrt{\frac{1}{16 \sigma^{4}}+T_{e}^{2}}\right)\right]^{1 / 2} \\
\cos \phi_{t}=\frac{1-1 / \sigma}{\Delta_{t}}, \quad \sin \phi_{t}=\frac{T_{e}}{\Delta_{t}} \\
\cos \phi_{c}=\frac{(1 / \varepsilon)(1 / \varepsilon-1 / \sigma)}{\Delta_{c}}, \quad \sin \phi_{c}=\frac{T_{e}}{\Delta_{c}} \\
\cos \phi_{i}=\frac{1 /(2 \sigma)+a+\Lambda \sqrt{\mathscr{H} / \Pi(1)}}{\Delta_{i}}, \quad \sin \phi_{i}=\frac{b}{\Delta_{i}}, \\
\Delta_{t}=\left[(1-1 / \sigma)^{2}+T_{e}^{2}\right]^{1 / 2}, \quad \Delta_{c}=\left[((1 / \varepsilon)(1 / \varepsilon-1 / \sigma))^{2}+T_{e}^{2}\right]^{1 / 2}, \\
\Delta_{i}=\left[\left(\frac{1}{2 \sigma}+a+\Lambda \sqrt{\frac{\mathscr{H}}{\Pi(1)}}\right)^{2}+b^{2}\right]^{1 / 2} .
\end{gathered}
$$

The parameter $T_{e}$ is defined as

$$
T_{e} \equiv(-1)^{n} T_{a p}^{1 / 2} C_{n}+\frac{1}{2}(-1)^{n_{s}} T_{a s}^{1 / 2} .
$$

With this parameter the combined effect due to spin and precession can be expressed as a general trend, as will be shown in $\S 3.2$.

Equation (3.19) illustrates that the flow in the fluid layer varies periodically with time by a frequency equal to the spin angular velocity $\Omega$, similar to that of the mushy layer. The velocity also varies in the vertical direction and consists of three parts differentiated by length scale. The first part is of the length scale of the thermal boundary layer, varying vertically with the exponential function $\mathrm{e}^{-\left(z-h_{b}\right)}$, and will be called the thermal-layer flow. The second part is of the length scale of the solute boundary layer, varying with $\mathrm{e}^{-\left(z-h_{b}\right) / \varepsilon}$, and will be called the solute-layer flow. The third part is of the length scale of the Ekman layer, varying with $\mathrm{e}^{-\left(z-h_{b}\right) / d_{E}}$ and changing direction with a period $2 \pi / b$, and will be called the Ekman-layer flow. A larger speed of rotation (in terms of larger $T_{e}$ ) leads to a larger $b$ and thus a smaller period of turnaround of the Ekman-layer flow.

To more clearly illustrate these three components of the induced flow in the fluid layer, we show in figure 2 their distributions with tank height for the case $T_{a p}=10^{6}$, $T_{a s}=0$, and $\phi_{n}=10^{\circ}$. It is seen from figure $2(a)$ that the velocities of both the thermallayer flow and the Ekman-layer flow decrease exponentially with height. The velocity of the solute-layer flow remains virtually the same except in the very-shallow solute boundary layer above the interface, in which the velocity also decreases (although very little) exponentially with height; it therefore makes very little contribution to the induced flow, or the combination of these three components. In figure $2(b)$ one can see that neither the thermal-layer flow nor the solute-layer flow changes direction while the direction of the Ekman-layer flow changes periodically with height. More precisely, near the fluid/mush interface, the direction of the Ekman-layer flow is virtually opposite to that of the thermal-layer flow, so that the induced flow velocity is small. At $z \approx 1.12$ these two flows are in the same direction, so that the induced flow has the largest velocity. Beyond this point, the thermal-layer flow dominates the induced flow. 
(a)

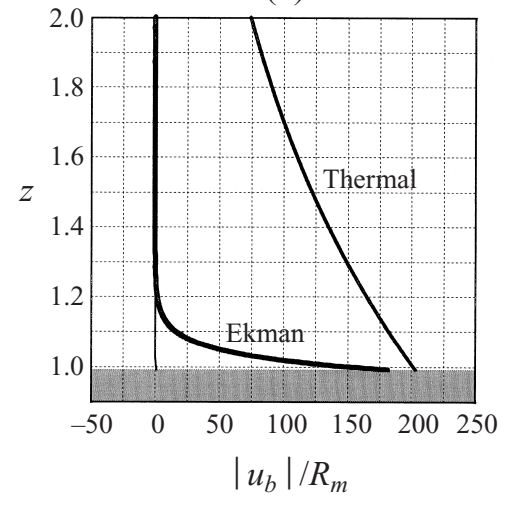

(b)

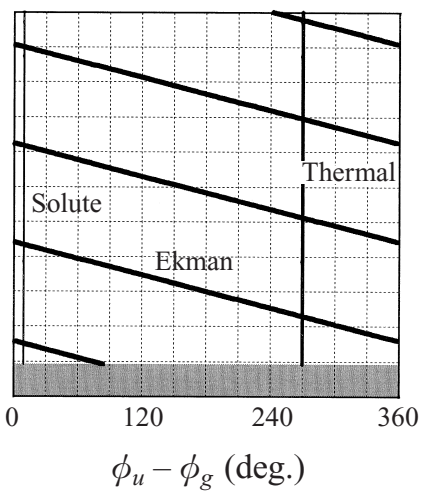

FIGURE 2. (a) The velocity distributions along the $z$-axis of the thermal-layer flow, the Ekman-layer flow, and the solute-layer flow. The velocities of both the thermal-layer and the Ekman-layer flows decrease exponentially with height, while that of solute-flow remains virtually the same in the whole fluid layer except in the shallow solute boundary layer above the interface, in which it also decreases (although very little) with height. (b) The phase-angle variations with height of these three flows. The phase angles of both the thermal-layer and the solute-layer flows remain the same over the whole fluid layer (the two vertical lines) and that of the Ekman-layer changes periodically with height with period $2 \pi / b$.

The coefficients of the velocity functions of (3.15) and (3.19) contain either $S_{n} R_{m}, S_{n} R_{t}$, or $S_{n} R_{c}$, indicating that the induced flow is a result of the imbalance between gravity and the pressure gradient in the $(x, y)$-plane when the angle between gravity and the density gradient is greater than zero. Accordingly, when the system rotates with a vertical axis, there is no induced flow ( $\mathrm{Lu} \&$ Chen 1997). Otherwise, flow is induced by the components of both the pressure gradient and gravity in the $(x, y)$-plane to move along the melt/mush interface (or the $(x, y)$-plane according to the present coordinate system). Because of the inclined spin, the $(x, y)$-plane components of both the pressure gradient and gravity change direction periodically with frequency $\Omega$. Consequently, the induced flows in both the fluid and mushy layers are periodic functions of $\Omega$, see (3.15) and (3.19). This however does not apply to the precession-only case because

$$
g_{x}+\mathrm{i} g_{y}=\left(S_{n} g\right) \exp \left(\mathrm{i} \phi_{g}\right) .
$$

By comparing (3.22) with the exponential terms of (3.15) and (3.19), one can see that the phase-angle difference between the induced flow and gravity is independent of time, implying that the induced flow due to inclination is steady in the precession-only case.

\subsection{Examples of induced flow}

To illustrate more concretely the induced flow due to the inclination, we compute (3.15) and (3.19) for the $26 \%$ ammonium chloride solution, whose physical properties can be obtained from Chen et al. (1994). Note that according to the growth rate shown in Chen, Yang \& $\mathrm{Lu}$ (1993), $T_{a p}=10^{6}$ (or $T_{a s}=10^{6}$ ) is equivalent to the angular velocity 0.7 r.p.m., and $T_{a p}=10^{8}$ (or $T_{a s}=10^{8}$ ) to 7 r.p.m. A Taylor number within this range was considered in the experiment of Sample \& Hellawell (1984), and accordingly will also be considered in the following analysis. We assume also 

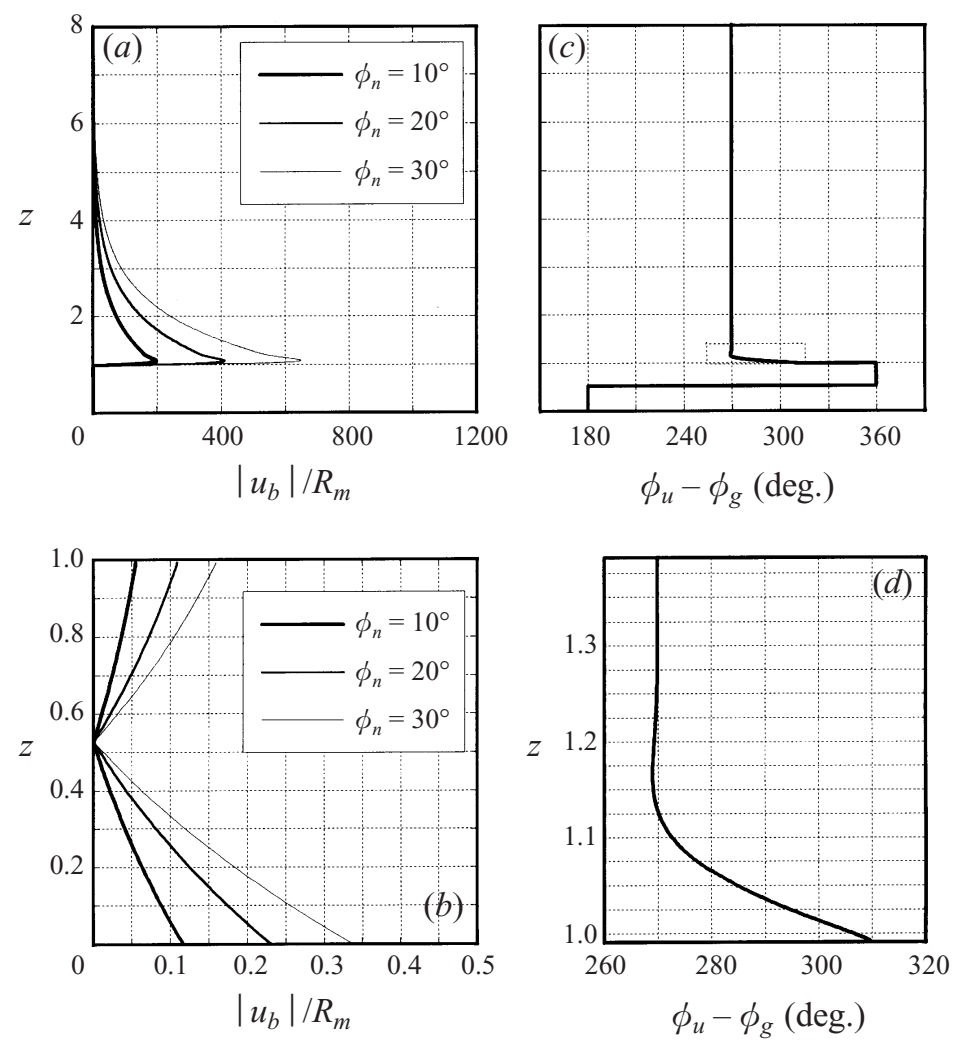

FiguRE 3. The induced flow in both the fluid and the mushy layers for a $26 \mathrm{wt} \%$ ammonium chloride solution when $T_{a p}=10^{6}, T_{a s}=0$, and $n_{p}=0$. (a) The induced flow in the fluid layer. (b) The induced flow in the mushy layer. $(c)$ The phase angle difference between the induced flow and the gravity component in the $(x, y)$-plane. $(d)$ An enlargement of the curve enclosed by the dotted rectangle in $(c)$, where one can see that the direction of the induced flow in the Ekman layer changes by about $40^{\circ}$ or more. In $(a)$ and $(b)$ three inclination angles are shown, illustrating that larger inclination angle leads to a larger induced velocity.

that the mush is of homogeneous permeability $\Pi_{b}=1$, as did in Worster (1992) and Chen et al. (1994).

Figure 3 illustrates the case $T_{a p}=10^{6}$ and $T_{a s}=0$; the system rotates with precession only. The velocity distribution shown in figure $3(a)$ indicates that most of the induced flow occurs in the fluid layer while that in the mush is relatively much smaller and virtually cannot be seen in the figure. In the fluid layer, the induced velocity increases sharply with height from the melt/mush interface, reaches a maximum within a short distance and then decreases exponentially into the bulk melt. The sharp velocity gradient is confined to the Ekman layer, whose thickness is of $O\left(d_{E}\right)$, where $d_{E} \approx\left(\left|T_{e}\right| / 2\right)^{-1 / 2}=0.045$. The Ekman layer is very thin compared with the thermal boundary layer whose thickness is of $O(1)$, while it is larger than the solute boundary layer whose thickness is of $O(\varepsilon)$, where $\varepsilon=O\left(10^{-2}\right)$ for most of the aqueous solutions. In the mushy layer (figure $3 b$ ), due to the high resistance to the flow, the induced velocity is of $O\left(10^{-1}\right)$, much smaller than that of the fluid layer, which is of $O\left(10^{2}\right)$, and also increases with inclination angle. We note that the mushy layer is separated into two regions in terms of flow direction. One lies in $0<z<0.525$, where the phase angle of the velocity $\left(\phi_{u}\right)$ differs from that of gravity 

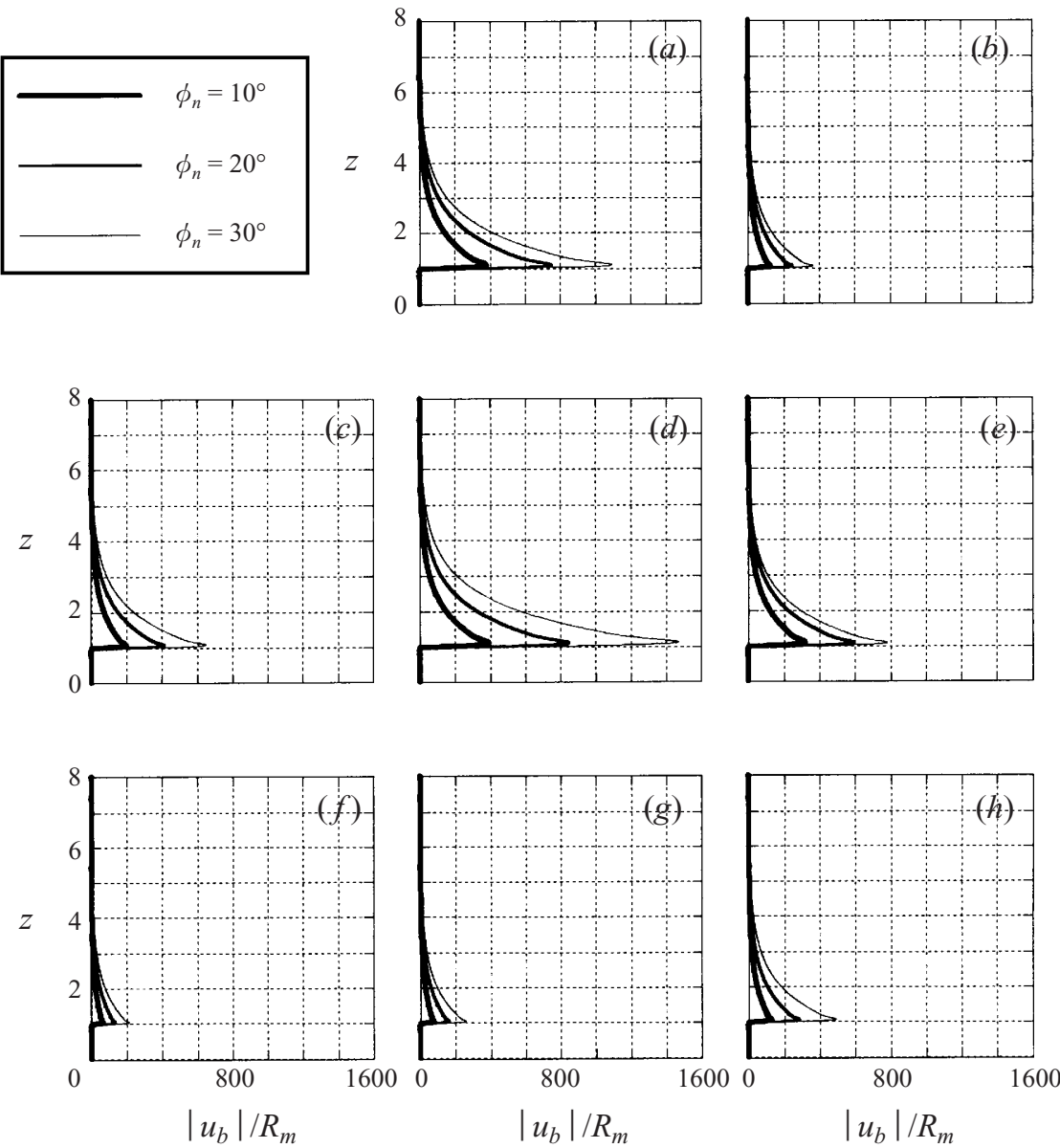

FIGURE 4. The induced flow for various $T_{a s}$ and $T_{a p}$ when the spin and precession are in the opposite sense of rotation, i.e. $n_{S}=0$ and $n_{p}=1$. In each plot three curves corresponding to different $\phi_{n}$ are shown, whereas the velocity in the mush is too small to be seen. From left to right, the three columns are respectively for $T_{a s}=0,10^{6}$ and $10^{7}$. From top to bottom, the three rows are respectively for $T_{a p}=0,10^{6}$ and $10^{7}$. Note that the trend of the change of induced flow with $T_{a s}$ and $T_{a p}$ is not straightforward. A new parameter, the effective Taylor number $T_{e}$ defined in (3.21), is employed to account for the rotation effect in a general sense, as will be shown in figure 5 .

$\left(\phi_{g}\right)$ by $180^{\circ}$ (figure $3 c$ ), suggesting that along the interface the pressure gradient is larger than gravity so that the flow moves upwards along the interface. The other region lies in $0.525<z<h_{b}$, where the pressure gradient is smaller than gravity, and the flow moves downwards along the interface. Note that the mushy-layer depth for the $26 \%$ ammonium chloride solution is obtained by substituting (3.6) into (3.7), yielding $h_{b}=0.991$. At the melt/mush interface, due to the use of $(3.14 a, b)$, there is a difference between the flow velocities in the fluid and the mushy layers, and so there is a difference between the phase angles of the two flows. More precisely, $\phi_{u}-\phi_{g}$ changes from $360^{\circ}$ in the mushy layer to $310^{\circ}$ in the fluid layer (see figures $3 c$ and $3 d$ ).

It is also seen from figure $3(c)$ that, due to the slow motion in the mush, the Coriolis force in the mush is negligible so that the flow direction in the mush does not change with height. In the fluid layer, however, the flow direction in the Ekman layer changes with height dramatically (figure $3 d$ ) : $\phi_{u}-\phi_{g}$ decreases from about $310^{\circ}$ to about $270^{\circ}$ 


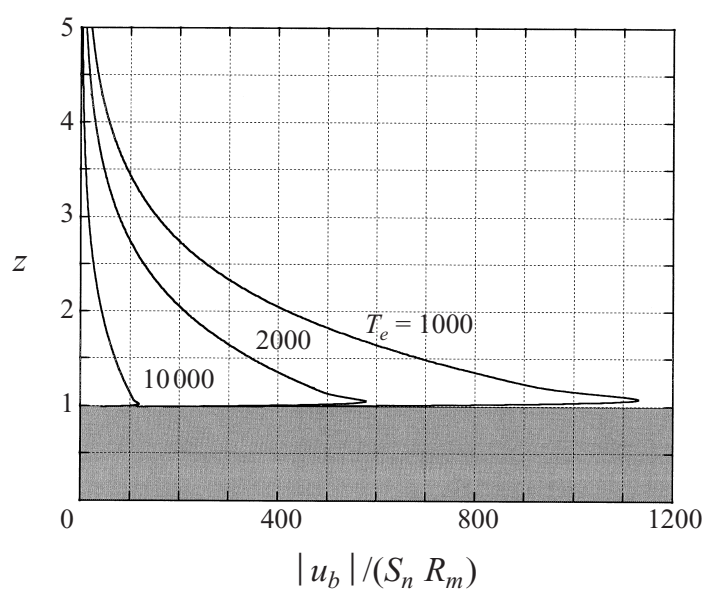

FIGURE 5. The velocity profiles of the induced flow corresponding to three different $T_{e}$. Note that a larger $T_{e}$ leads to a smaller induced velocity.

within the Ekman layer and then remains virtually the same in the thermal boundary layer. As a result, the flow in the thermal boundary layer, which extends to the far field, is in a direction leading gravity by $90^{\circ}$ (i.e. $\phi_{u}-\phi_{g}=270^{\circ}$ ), and this applies to all the cases considered in the present paper.

To investigate the combined effect of spin and precession on the induced flow, we illustrate in figure 4 cases in which spin and precession are of opposite sense of rotation, i.e. $n_{s}=0$ and $n_{p}=1$. The plots from left to right are respectively for $T_{a s}=0,10^{6}$ and $10^{7}$, and those from top to bottom are respectively for $T_{a p}=0,10^{6}$ and $10^{7}$. The trend of the combined effect is not straightforward. The maximum velocity occurs when $T_{a s}=10^{6}$ and $T_{a p}=10^{6}$ (figure $4 d$ ) and the minimum velocity occurs when $T_{a s}=0$ and $T_{a p}=10^{7}$ (figure $4 f$ ). To illustrate the combined effect due to spin and precession in a more systematic way, we employ the effective Taylor number $T_{e}$ given in (3.21). As shown in figure 5 , the induced flow velocity decreases with increasing $T_{e}$, suggesting that the induced flow is inhibited by higher rotation speed, which is explained as follows. It is known that the induced flow results from the interaction among the pressure gradient, gravity and the Coriolis force. As the inclination angle is fixed, both the pressure gradient and gravity are then fixed. The Coriolis force will accordingly be fixed also, to keep these three external forces balanced. Since the Coriolis force is the product of induced flow velocity and the rotation speed, as the rotation speed increases the induced velocity must decrease.

\subsection{Limits to the validity of the induced-flow solution}

The derivation of the induced flow of $\S 3.1$ is made under the assumption that the dimensions of the cooling tank (or the system) in both the vertical and the horizontal directions are much larger than the characteristic length of solidification $H$, so that both the nonlinear advection term and the diffusion term in the horizontal direction can be neglected and the reduced equations can be solved exactly. In other words, the dimensions of the cooling tank present limitations, which are different for the systems under spinning and under precession because the intrinsic features of the induced flow of these two cases are different. In this section, we will discuss for the two cases of precession only and spin and precession the limitations due to the cooling tank 
dimension so that the requirements for the existence of the induced flow shown in $\S 3.1$ can be determined.

\subsubsection{Precession-only case}

Although there is a precession angular velocity $\dot{\phi}_{p}$ in the system, it does not appear with $t$ in the same term of the governing equations because, as explained in the previous section, the direction of gravity with respect to the cooling tank remains the same during precession. Accordingly, the time scale of solidification $H / V$ still dominates the system. The unsteady terms in the governing equations are therefore of the same order of magnitude as the terms due to interface growth (i.e. the term containing $\partial / \partial z$ ). Consequently, due to the assumptions leading to the requirements described by (3.1) and (3.4), the horizontal dimension of the cooling tank must satisfy both of these two conditions. Based on these two conditions, we make a scale analysis for (3.19), considering the case for $26 \mathrm{wt} \%$ ammonium chloride solution, yielding

$$
U / V \propto S_{n} R_{t} / \Delta_{t} .
$$

By substituting it into (3.4) and considering the precession-only case (i.e. $T_{a s}=0$ ), we obtain

$$
\frac{L}{H} \gg \frac{S_{n} R_{t}}{\left[(1-1 / \sigma)^{2}+T_{a p} C_{n}^{2}\right]^{1 / 2}} .
$$

For a tank containing $26 \%$ ammonium chloride solution precessing with an angular velocity 1 r.p.m. around an axis of inclination angle $20^{\circ}-30^{\circ}$, the physical parameters in (3.24) are of the following order of magnitude: $R_{t}=O\left(10^{7}\right), \sigma=O(10)$ and $T_{a p}=O\left(10^{6}\right)$. In such a system, according to (3.24), the dimension of the tank requires $L / H \gg O\left(10^{4}\right)$, which is more restrictive than $(3.1)$. Since $H \approx 1 \mathrm{~cm}$, the horizontal dimension of the tank is required to be much larger than the normal scale considered in either industry or laboratory. The tank used by Sample \& Hellawell (1984), for example, had horizontal radius $7.5 \mathrm{~cm}$ and height is $16 \mathrm{~cm}$, which do not satisfy the requirements, so that the induced flow shown in $\S 3.1$ does not exist in their system. A spiral motion may instead occur because of the blockage to the induced flow imposed by the sidewalls of the tank. We however note that a small inclination angle and/or a high precession angular velocity may allow a smaller dimension of the tank. For example, for a system precessing by 10 r.p.m. with respect to an axis of inclination angle equal to $5^{\circ}$, the requirement of (3.24) becomes $L / H \gg O\left(10^{2}\right)$, which is more acceptable.

The requirement for the height of the tank, $H_{t}$ should be that it is much larger than the depths of both the thermal and the solute boundary layers:

$$
\frac{H_{t}}{H} \gg 1 \text {. }
$$

Equation (3.25) applies also to the condition that requires the satisfaction of rigid body rotation of the fluid at far field.

\subsubsection{Precession-and-spin case}

As the tank is spinning (with or without precession) with respect to an inclined axis, the direction of gravity changes periodically with frequency equal to the spin angular velocity $\dot{\phi}_{s}$. As a result, the characteristic time scale of the system is $\dot{\phi}_{s}^{-1}$. If the Strouhal number of spin is large, namely, $\dot{\phi}_{s} L / U \gg 1$, this can be rewritten by 
dividing both side by $H$ and by employing the definition of $(2.18 b)$ :

$$
\frac{L}{H} \gg \frac{U}{\dot{\phi}_{s} H}=\frac{2 U}{\sigma T_{a s}^{1 / 2} V} .
$$

The nonlinear advection term can thus be neglected compared with the unsteady term. It is much easier to satisfy (3.26) than (3.4) because generally $T_{a s} \gg 1$. Consequently, the horizontal dimension of the cooling tank can be determined by the following condition:

$$
\frac{L}{H} \gg \frac{2 S_{n} R_{t}}{\sigma T_{a s}^{1 / 2}\left[(1-1 / \sigma)^{2}+T_{e}^{2}\right]^{1 / 2}},
$$

which is obtained by substituting (3.23) into (3.26). For a tank containing a $26 \%$ ammonium chloride solution spinning with a speed of 1 r.p.m. with an inclination angle of $20^{\circ}-30^{\circ}$, the requirement of (3.27) is $L / H \gg O(1)$, which is similar to the requirement of (3.1). For a higher $\dot{\phi}_{s}$, the requirement of (3.27) will become less strict than (3.1).

As we have shown previously, when the tank spins with an inclined axis, the induced flow changes direction with a frequency equal to the spin angular velocity $\Omega$ (or equivalently $\dot{\phi}_{s}$ ). The projection of a fluid-particle trajectory on the $(x, y)$-plane is a circle and the radius of the circle is equal to the right-hand side of (3.26). Equation (3.26) requires that the horizontal dimension of the tank be much larger than the radius of the trajectory circle, which is about the same order of magnitude as the characteristic length of solidification (or the mushy-layer depth). In the mush, due to the fact that the velocity of the induced flow is much smaller than that of the fluid layer, the radius of the trajectory circle will be much smaller than the depth of the mush.

The requirement for the height of the tank due to inclined spin is considered in the following. To satisfy the rigid-body rotation condition at the far field, or to ignore the effect of the possible occurrence of a gravity wave on the free surface, the height of the tank must be much larger than its horizontal dimension, namely

$$
H_{t} \gg L \text {. }
$$

Together with the requirement of (3.1) and (3.26), the sufficient condition for the existence of the induced flow for the spin case (with or without precession) is $H_{t} \gg L \gg H$. For the experiment with $26 \%$ ammonium chloride solution, $H \approx 1 \mathrm{~cm}$, so that the cooling tank used by Sample \& Hellawell (1984) would satisfy the requirement.

\section{Linearized perturbation equations in the mush}

In implementing the linear stability analysis for the flow of $\S 3$, there are at least two essential numerical difficulties in solving these equations. First, since the induced flow changes direction with height, it is necessary to consider the instability modes corresponding to all the directions from $0^{\circ}$ to $360^{\circ}$ (in fact due to the complex conjugation only $0^{\circ}$ to $180^{\circ}$ needs to be considered). The scale of the computation is therefore extremely big. Additionally, since the coefficients of the perturbed equations and boundary conditions are functions of time, one needs to employ the Floquet theory (see Murray et al. 1993) to deal with the time-periodic coefficients. Use of Floquet theory, increases the order of differentiation of the equations from fifteen to 
$30 L+15$, where $L$ is the number of the terms of the Fourier time series. For a medium Taylor number, $L \geqslant 5$ is quite usual.

To render the linear stability analysis numerically tractable, it is necessary to reduce the order of the differentiation. The way to achieve this goal is to focus on the stability in the mushy layer only, as done by, for example, Amberg \& Homsy (1993), Anderson \& Worster (1995). The main reason for their focusing on the stability in the mushy layer is that the convection in the mush eventually leads to the formation of chimneys (or freckles). They conjectured also that the characteristics of the convection in the mush are hardly influenced by the flow in the fluid layer. This conjecture has been confirmed by Emms \& Fowler (1994) who showed that the vigorous salt-finger convection above the fluid/mush interface has a small effect on the convection in the mush.

To derive the linear equations of the mushy layer, we introduce small perturbation quantities together with the basic state into (2.14) and neglect the products of small quantities. After eliminating the pressure and the velocities in both the $x$ - and $y$ directions, the small perturbation equations in the mushy layer are

$$
\begin{gathered}
\left(\frac{\partial}{\partial t}-\frac{\partial}{\partial z}+u_{b} \frac{\partial}{\partial x}+v_{b} \frac{\partial}{\partial y}-\nabla^{2}\right) \theta+\mathscr{F}\left(\frac{\partial}{\partial t}-\frac{\partial}{\partial z}\right) \chi=-\theta_{b}^{\prime} w \\
{\left[\chi_{b}\left(\frac{\partial}{\partial t}-\frac{\partial}{\partial z}\right)+u_{b} \frac{\partial}{\partial x}+v_{b} \frac{\partial}{\partial y}-\chi_{b}^{\prime}\right] \theta+\left[\left(\theta_{b}-C\right)\left(\frac{\partial}{\partial t}-\frac{\partial}{\partial z}\right)-\theta_{b}^{\prime}\right] \chi=-\theta_{b}^{\prime} w} \\
\left\{\frac{\nabla^{2}}{\Pi_{b}}+\frac{S_{n}(-1)^{n_{p}} T_{m p}^{1 / 2} \chi_{b}^{\prime}}{\chi_{b}^{2}}\left[C_{s}(t) \frac{\partial}{\partial x}-S_{s}(t) \frac{\partial}{\partial y}\right]-\frac{\Pi_{b}^{\prime}}{\Pi_{b}^{2}} \frac{\partial}{\partial z}\right\} w \\
+\left\{S_{n}(-1)^{n_{p}} T_{m p}^{1 / 2}\left[S_{s}(t) \frac{\partial}{\partial x}+C_{s}(t) \frac{\partial}{\partial y}\right]+\left[C_{n}(-1)^{n_{p}} T_{m p}^{1 / 2}+(-1)^{n_{s}} T_{m s}^{1 / 2}\right] \frac{\partial}{\partial z}\right\}\left(\frac{\varsigma}{\chi_{b}}\right) \\
-\left[C_{n}(-1)^{n_{p}} T_{m p}^{1 / 2}+(-1)^{n_{s}} T_{m s}^{1 / 2}\right] \frac{\partial}{\partial z}\left[\frac{1}{\chi_{b}^{2}}\left(v_{b} \frac{\partial}{\partial x}-u_{b} \frac{\partial}{\partial y}\right) \chi\right] \\
+\frac{S_{n}(-1)^{n_{p}} T_{m p}^{1 / 2}}{\chi_{b}^{2}}\left[C_{s}(t) u_{b}-S_{s}(t) v_{b}\right] \nabla_{H}^{2} \chi+\frac{\partial}{\partial z}\left(\frac{u_{b}}{\Pi_{b}^{2}} \frac{\partial}{\partial x}+\frac{v_{b}}{\Pi_{b}^{2}} \frac{\partial}{\partial y}\right) \Pi \\
\varsigma=-R_{m}\left\{C_{n} \nabla_{H}^{2}-S_{n}\left[S_{s}(t) \frac{\partial}{\partial x}+C_{s}(t) \frac{\partial}{\partial y}\right] \frac{\partial}{\partial z}\right\} \theta \\
\quad \frac{\Pi_{b}}{\chi_{b}}\left\{S_{n}(-1)^{n_{p}} T_{m p}^{1 / 2}\left[S_{s}(t) \frac{\partial}{\partial x}+C_{s}(t) \frac{\partial}{\partial y}\right]+\left[C_{n}(-1)^{n_{p}} T_{m p}^{1 / 2}+(-1)^{n_{s}} T_{m s}^{1 / 2}\right] \frac{\partial}{\partial z}\right\} w \\
+\left[C_{n}(-1)^{n_{p}} T_{m p}^{1 / 2}+(-1)^{n_{s}} T_{m s}^{1 / 2}\right] \frac{\Pi_{b}}{\chi_{b}^{2}}\left(u_{b} \frac{\partial}{\partial x}+v_{b} \frac{\partial}{\partial y}\right) \chi \\
+\left(v_{b} \frac{\partial}{\partial x}-u_{b} \frac{\partial}{\partial y}\right)\left(\frac{\Pi}{\Pi_{b}}\right)-S_{n} R_{m} \Pi_{b}\left[C_{s}(t) \frac{\partial}{\partial x}-S_{s}(t) \frac{\partial}{\partial y}\right] \theta
\end{gathered}
$$

In the above equations, $\theta$ is temperature (or equivalently concentration), $w$ and $\varsigma$ are respectively the velocity and the vorticity in the vertical direction, $\chi$ is the porosity, and $\Pi$ is the permeability. All these are small perturbation quantities. Also, $\theta_{b}, u_{b}, v_{b}, \chi_{b}$ and $\Pi_{b}$ are respectively the temperature, the $x$-direction velocity, the $y$ direction velocity, the porosity, and the permeability of the basic state as shown in $\S 3$. 
The prime denotes differentiation with respect to $z$. The planar Laplacian operator $\nabla_{H}^{2}$ is defined as $\nabla_{H}^{2}=\partial^{2} / \partial x^{2}+\partial^{2} / \partial y^{2}$. In the following analysis we assume that the permeability is a uniform constant, i.e. $\Pi_{b}=1$ and $\Pi=0$ (Worster 1992; Chen et al. 1994) to further simplify the problem.

Equations (4.1) consists of five orders of differentiation and needs accordingly five boundary conditions. The perturbed boundary conditions at the solid/mush interface $z=0$ are

$$
\theta=0, \quad w=0 .
$$

At the fluid/mush interface $z=h_{b}$, the conditions are

$$
\theta=0, \quad \chi=0 \text {. }
$$

These result from the assumptions that the mushy-layer height is constant, the porosity is unity, and the temperature is fixed at the value of the basic state. Consequently, the perturbations of both the temperature and porosity vanish. Regarding the velocity at $z=h_{b}$, we consider the pressure to be fixed at the basic state such that along the interface the perturbation of pressure also vanishes, i.e.

$$
p=0 .
$$

Imposing a small perturbation on the induced velocity, (3.15), and substituting this into $(2.14 b, c)$, yields the perturbed momentum equations in both the $x$ - and $y$-directions as

$$
\begin{gathered}
\frac{u}{\Pi_{b}}-\frac{\left\lfloor C_{n}(-1)^{n_{p}} T_{m p}^{1 / 2}+(-1)^{n_{p}} T_{m s}^{1 / 2}\right\rfloor}{\chi_{b}} v+\frac{S_{n}(-1)^{n_{p}} T_{m p}^{1 / 2} C_{s}(t)}{\chi_{b}} w \\
+\frac{\left[C_{n}(-1)^{n_{p}} T_{m p}^{1 / 2}+(-1)^{n_{s}} T_{m s}^{1 / 2}\right] v_{b}}{\chi_{b}^{2}} \chi=-R_{m}\left[\frac{\partial p}{\partial x}+S_{n} S_{s}(t) \theta\right], \\
\frac{v}{\Pi_{b}}+\frac{\left\lfloor C_{n}(-1)^{n_{p}} T_{m p}^{1 / 2}+(-1)^{n_{s}} T_{m s}^{1 / 2}\right\rfloor}{\chi_{b}} u-\frac{S_{n}(-1)^{n_{p}} T_{m p}^{1 / 2} S_{s}(t)}{\chi_{b}} w \\
-\frac{\left[C_{n}(-1)^{n_{p}} T_{m p}^{1 / 2}+(-1)^{n_{s}} T_{m s}^{1 / 2}\right] u_{b}}{\chi_{b}^{2}} \chi=-R_{m}\left[\frac{\partial p}{\partial y}+S_{n} C_{s}(t) \theta\right] .
\end{gathered}
$$

For the case of $26 \mathrm{wt} \%$ ammonium chloride solution and moderate rotation speed, the Taylor number in the mush is very low, i.e.

$$
T_{m p} \ll 1, \quad T_{m s} \ll 1 .
$$

The Coriolis force in the mush is accordingly negligible. As a result, $(4.5 a, b)$ can be further simplified as

$$
\frac{u}{\Pi_{b}} \approx-R_{m}\left[\frac{\partial p}{\partial x}+S_{n} S_{s}(t) \theta\right], \quad \frac{v}{\Pi_{b}} \approx-R_{m}\left[\frac{\partial p}{\partial y}+S_{n} C_{s}(t) \theta\right] .
$$

By substituting (4.3a) and (4.4) into $(4.7 a, b)$, one obtains at $z=h_{b}$

$$
u_{-} \approx 0, \quad v_{-} \approx 0,
$$

where the index - denotes under the interface. After substituting $(4.8 a, b)$ into the continuity equation, we obtain at $z=h_{b}$

$$
\frac{\partial w_{-}}{\partial z} \approx 0
$$


Consequently, the boundary conditions at the fluid/mush interface $\left(z=h_{b}\right)$ are $(4.3 a, b)$ and (4.9), and at the mush/solid interface $(z=0)$ they are $(4.2 a, b)$, and the governing equations of the mush are (4.1). These equations constitute the mathematical model governing the stability of the flow in the mush.

\section{Linear stability analysis I: precession-only case}

In this section the linear stability analysis in the mushy layer is implemented for the precession-only case. The governing equation is (4.1) and the boundary conditions are $(4.2 a, b),(4.3 a, b)$ and (4.9). The basic flow is given by (3.15), which, as shown in figure $3(b)$, is a parallel flow consisting of two separated flows of opposite directions. Since the coefficients of (4.1) are functions of $z$ only, we can apply the normal mode expansion to the dependent variables

$$
\left[\begin{array}{l}
\theta(x, y, z, t) \\
w(x, y, z, t) \\
\varsigma(x, y, z, t) \\
\chi(x, y, z, t)
\end{array}\right]=\left[\begin{array}{l}
\hat{\theta}(z) \\
\hat{w}(z) \\
\hat{\varsigma}(z) \\
\hat{\chi}(z)
\end{array}\right] \exp \left[\omega t+\mathrm{i}\left(k_{x} x+k_{y} y\right)\right]+\text { c.c. }
$$

By substituting (5.1) into (4.1), we obtain the following ordinary differential equations:

$$
\begin{gathered}
\left(\mathrm{D}^{2}+\mathrm{D}-\omega-k^{2}-\mathrm{i} k_{y} v_{b}\right) \hat{\theta}+\mathscr{F}(\mathrm{D}-\omega) \hat{\chi}=\theta_{b}^{\prime} \hat{w} \\
{\left[\chi_{b}(\mathrm{D}-\omega)+\chi_{b}^{\prime}-\mathrm{i} k_{y} v_{b}\right] \hat{\theta}+\left[\left(\theta_{b}-\mathscr{C}\right)(\mathrm{D}-\omega)+\theta_{b}^{\prime}\right] \hat{\chi}=\theta_{b}^{\prime} \hat{w}} \\
\left(\mathrm{D}^{2}-k^{2}+\frac{\mathrm{i} k_{x} S_{n} T_{m p}^{1 / 2} \chi_{b}^{\prime}}{\chi_{b}^{2}}\right) \hat{w}+T_{m p}^{1 / 2}\left(\mathrm{i} k_{y} S_{n}+C_{n} \mathrm{D}\right)\left(\frac{\hat{\varsigma}}{\chi_{b}}\right)-\mathrm{i} k_{x} C_{n} T_{m p}^{1 / 2} \mathrm{D}\left(\frac{v_{b} \chi}{\chi_{b}^{2}}\right) \\
=R_{m}\left(C_{n} k^{2}+\mathrm{i} k_{y} S_{n} \mathrm{D}\right) \hat{\theta} \\
\hat{\varsigma}=\frac{T_{m p}^{1 / 2}}{\chi_{b}}\left(\mathrm{i} k_{y} S_{n}+C_{n} \mathrm{D}\right) \hat{w}+\frac{\mathrm{i} k_{y} C_{n} T_{m p}^{1 / 2} v_{b}}{\chi_{b}^{2}} \chi-\mathrm{i} k_{x} S_{n} R_{m} \theta
\end{gathered}
$$

where $\mathrm{D}=\mathrm{d} / \mathrm{d} z$. The boundary conditions at $z=h_{b}$ are

$$
\hat{\theta}=0, \quad \hat{\chi}=0, \quad \mathrm{D} \hat{w}=0,
$$

and at $z=0$ are

$$
\hat{\theta}=0, \quad \hat{w}=0 .
$$

Equations (5.2), (5.3) and (5.4) constitute an complex eigenvalue problem

$$
F=F\left(R_{m}, k, \omega ; S_{n}, \phi_{k}, T_{m p}, \mathscr{F}, \mathscr{C}, \mathscr{A}, \theta_{\infty}\right),
$$

where $\phi_{k}$ is the angle between the direction of propagation of the disturbance and the $x$-axis, related to the horizontal wavenumbers $k_{x}$ and $k_{y}$ by

$$
k_{x}=k \cos \left(\phi_{k}\right), \quad k_{y}=k \sin \left(\phi_{k}\right) .
$$

To analyse (5.5), it is necessary to consider $0^{\circ} \leqslant \phi_{k} \leqslant 180^{\circ}$. Physically, this means that the stability characteristics of the disturbance change when the direction of propagation changes.

We consider two different models based on different assumptions: (1) small $\phi_{n}$ and (2) large $\phi_{n}$. For the small- $\phi_{n}$ model, the equations can be greatly simplified so that 

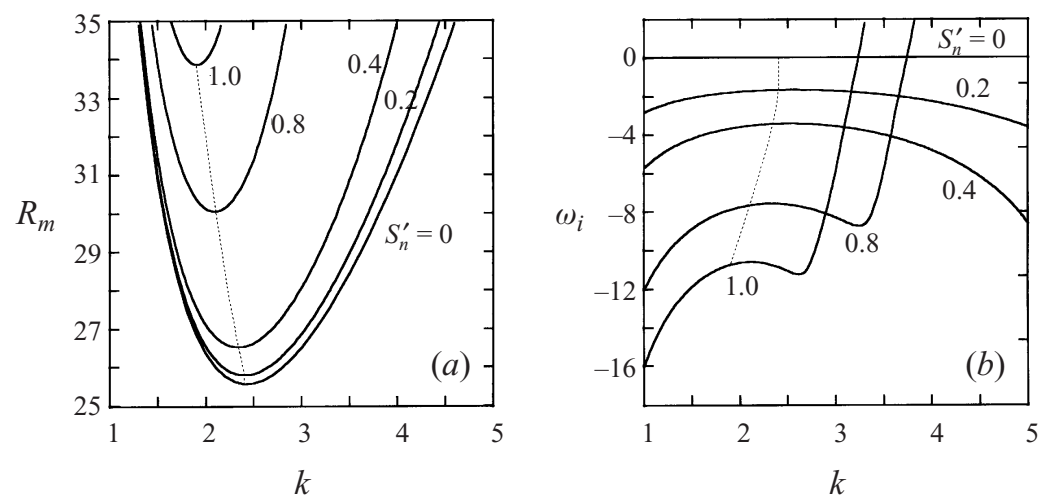

FIGURE 6. (a) The neutral curves and $(b)$ the oscillatory frequency $\omega_{i}$ obtained by the small- $\phi_{n}$ model for various $S_{n}^{\prime}$. The Coriolis force is neglected. All the instability modes of $S_{n}^{\prime}>0$ are of oscillatory nature, travelling in the $(x, y)$-plane with a wave speed proportional to $\omega_{i}$. The instability modes of $S_{n}^{\prime}=0$ are steady in nature.

the numerical integration can be implemented more efficiently and the stability of the flow in a wide range of physical parameters can be analysed. The validity of the results of the small- $\phi_{n}$ model can be checked by the results of the large- $\phi_{n}$ model. Although the computation process is more tedious, the analysis based on the large- $\phi_{n}$ model provides a more thorough picture of the stability of the flow under precession.

\subsection{Small- $\phi_{n}$ case}

Because $\phi_{n}$ is small we may assume

$$
C_{n} \approx 1 .
$$

Based on (5.7) and $T_{m p} \ll 1$, (5.2) can be simplified as

$$
\begin{gathered}
\left(\mathrm{D}^{2}+\mathrm{D}-\omega-k^{2}-\mathrm{i} k \bar{v}_{b}\right) \hat{\theta}+\mathscr{F}(\mathrm{D}-\omega) \hat{\chi}=\theta_{b}^{\prime} \hat{w}, \\
{\left[\chi_{b}(\mathrm{D}-\omega)+\chi_{b}^{\prime}-\mathrm{i} k \bar{v}_{b}\right] \hat{\theta}+\left[\left(\theta_{b}-\mathscr{C}\right)(\mathrm{D}-\omega)+\theta_{b}^{\prime}\right] \hat{\chi}=\theta_{b}^{\prime} \hat{w},} \\
\left(\mathrm{D}^{2}-k^{2}\right) \hat{w}=R_{m}\left(k^{2}+\mathrm{i} k S_{n}^{\prime} \mathrm{D}\right) \hat{\theta},
\end{gathered}
$$

in which

$$
\bar{v}_{b}=-S_{n}^{\prime} R_{m}\left(\mathscr{A} \theta_{\infty}+\theta_{b}\right), \quad S_{n}^{\prime}=S_{n} \sin \left(\phi_{k}\right),
$$

where $(5.9 \mathrm{~b})$ is the so-called Squire transformation. As shown in (5.9), the effect due to different $\phi_{k}$ is included in the parameter $S_{n}^{\prime}$, which is called the effective-sine of the inclined angle. By using this new parameter, there is no need to compute all the disturbances in the different propagation directions so that the scale of the computation is greatly reduced.

Note that the $\bar{v}_{b}$ of (5.8) is the component of the induced flow in the direction in which the disturbance propagates. It implies that, in the mush with a small- $\phi_{n}$ precession and having negligible Coriolis force, the disturbance is affected only by the component of the induced flow in the direction of the disturbance. Since in the present case the induced flow is in the $y$-direction, the disturbance in the $x$-direction is not influenced by the induced flow, which is consistent with the result of Ingersoll (1966), who considered the convective instabilities in plane Couette flow.

Equation (5.8) and boundary conditions (5.3) and (5.4) constitute a complex eigen- 

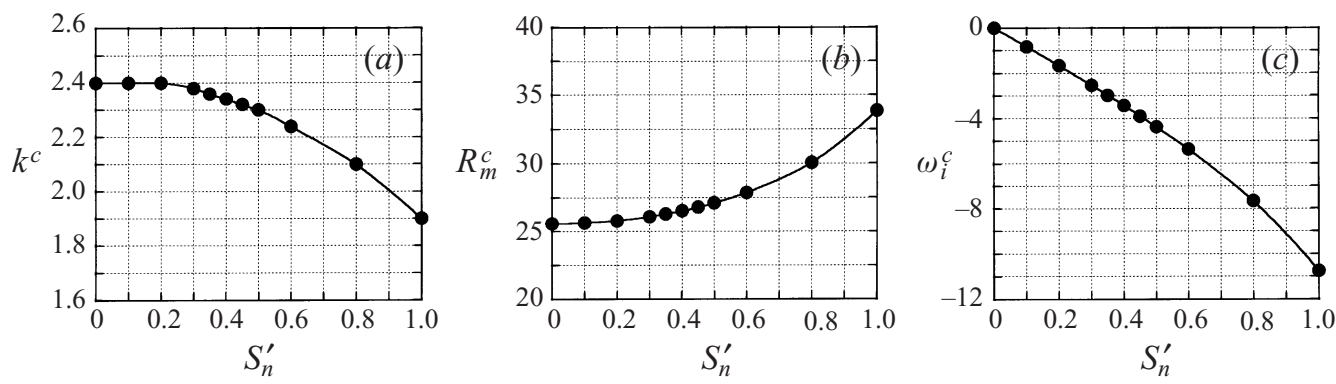

FiguRE 7. (a) The critical wavenumber $k^{c}$, (b) the critical Rayleigh number $R_{m}^{c}$, and (c) the critical oscillatory frequency $\omega_{i}^{c}$ corresponding to the case of figure 6 . It is seen that for a larger $S_{n}^{\prime}$, the most-unstable mode is of larger wavelength (inverse of wavenumber), greater stability, and faster travelling wave velocity.

value problem, which is solved by the shooting technique developed by Keller (1976), in which an orthonormalization process is applied to avoid the loss of linear independence of integration. The iteration procedure developed by Powell (1970) is employed to seek the convergence of the eigenvalues.

Figure 6 illustrates the neutral curves as well frequencies of the instability modes for various $S_{n}^{\prime}$. It is found that the instability modes are steady in nature and most unstable when $S_{n}^{\prime}=0$ (because $\omega_{i}=0$ ). The instability mode corresponding $S_{n}^{\prime}>0$ is more stable, being a wave travelling with a wave speed proportional to the oscillatory frequency $\omega_{i}$. The case of $S_{n}^{\prime}=0$ corresponds to two possibilities: (1) $\phi_{k}=0^{\circ}, 0^{\circ} \leqslant \phi_{n} \leqslant 90^{\circ}$ and (2) $\phi_{n}=0^{\circ}, 0^{\circ} \leqslant \phi_{k} \leqslant 180^{\circ}$, according to (2.8) and (5.9b). For the first possibility, the steady mode appears as a wave vector pointing in a direction perpendicular to the induced flow, which is the so-called longitudinal mode of instability. Physically, it means that for a system precessing with any inclination angle in the range $0^{\circ} \leqslant \phi_{n} \leqslant 90^{\circ}$, the most-unstable mode is the longitudinal mode. The modes propagating in the other directions are invariably more stable and thus will not occur normally. Similar phenomena were also found by, for example, Linden (1974) and Forth \& Wheeler (1992), in which double-diffusive convective flow is affected by the imposed shear flow, inducing a longitudinal instability mode, which turns out to be the most unstable one. For the second possibility, the system precessing around a vertical axis, the most-unstable mode may propagate without any preferred direction. In other words, the instability modes in different directions are of equal stability and steady in nature, as shown by Lu \& Chen (1997).

We plot in figure 7 the critical Rayleigh number $R_{m}^{c}$, the critical wavenumber $k^{c}$, and the corresponding oscillation frequency $\omega_{i}^{c}$ for various $S_{n}^{\prime}$. It is shown that the system is more stable for larger $S_{n}^{\prime}$. Since larger $S_{n}^{\prime}$ means either larger $\phi_{n}$ or larger $\phi_{k}$, the results therefore imply two possibilities in a physical sense: First, as shown in $\S 3$ and in the present section, larger $\phi_{n}$ leads to a larger induced flow and a more stable state, suggesting that the induced flow is stabilizing the system. Secondly, a larger $\phi_{k}$ means the induced flow (in the $y$-direction) has a larger component in the propagation direction, resulting in a more stable state. Meanwhile, the most-unstable mode is also of the largest wavenumber (or the smallest wavelength). As the system precesses with an axis of $\phi_{n}>0^{\circ}$ (inclined rotation), the instability mode in any direction behaves like a travelling wave. The wave speed (proportional to $\omega_{i}^{c}$ ) is higher for larger $S_{n}^{\prime}$. 

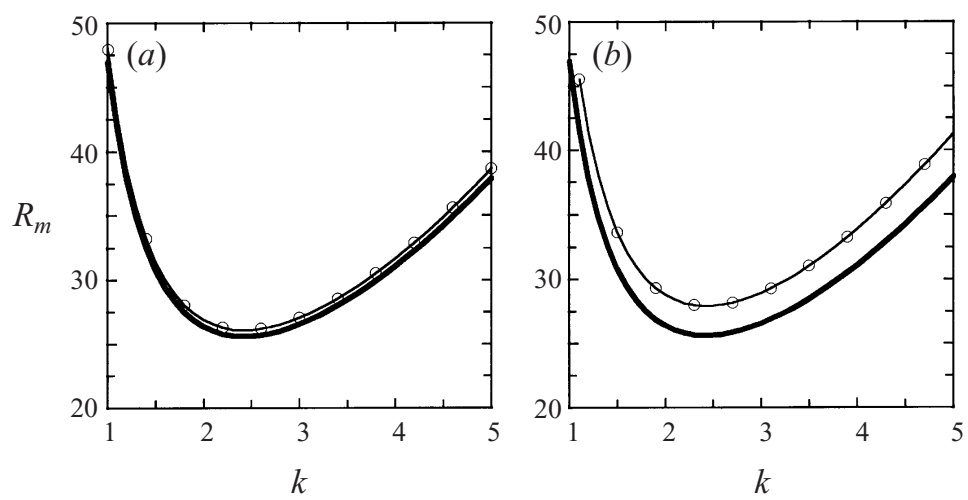

FIGURE 8 . The neutral curves obtained by the three different mathematical models. Heavy curve: small- $\phi_{n}$ model. Fine curve: large- $\phi_{n}$ model with Coriolis force effect. Fine curve with open circles: large- $\phi_{n}$ model without Coriolis force effect. (a) $S_{n}=0.2, \phi_{k}=0^{\circ} ;(b) S_{n}=0.4, \phi_{k}=0^{\circ}$.

\subsection{Large $-\phi_{n}$ case}

The assumption of small $\phi_{n}$ leads to the simplification that the buoyancy component in the direction of the solute gradient (or the $z$-direction) does not change with $\phi_{n}$, which to some extent does not reflect reality. This assumption will be lifted in this section so that the effect due to varying $\phi_{n}$ can be investigated. Meanwhile, due to the result shown in $\S 3$ that a larger $\phi_{n}$ leads to a larger induced flow and in turn results in a larger Coriolis force, we will thus in this section also study the effect of the Coriolis force on the stability of the system. The governing equation now is (5.2) and the boundary conditions are again (5.3) and (5.4).

For the case of large $\phi_{n}$ it is not feasible to apply the Squire transformation to (5.2). It is therefore necessary to look into the effect due to varying $\phi_{k}$. Figure 8 illustrates the neutral curves of the case $\phi_{k}=0^{\circ}$, and two inclination angles $S_{n}=0.2$ and $S_{n}=0.4$ are considered. In each plot, the heavy curve shows the results obtained from the small- $\phi_{n}$ model. The fine curve is the results of the large- $\phi_{n}$ model in which the Coriolis force, accounted for by a non-zero Taylor number $T_{a p}=10^{8}$ (or equivalently $\left.T_{m p}=8.2 \times 10^{-6}\right)$, is considered. The fine curve with open circles represents the results of the large- $\phi_{n}$ model when no Coriolis force is considered, i.e. $T_{a p}=0$. Results show that, first, for both the cases considered, the fine curve and the fine curve with circles virtually overlap each other, suggesting that the Coriolis force in the mush under moderate speed of precession does not influence the stability of the mush. Secondly, the $R_{m}$ of the large- $\phi_{n}$ model (fine curve) is invariably larger than that of the small- $\phi_{n}$ model (heavy curve), reflecting the fact that the reduction in buoyancy component in the $z$-direction stabilizes the system. This implies also that the neglect of the buoyancy reduction along the $z$-direction in the small- $\phi_{n}$ model can become crucial when $\phi_{n}$ is not small.

To elucidate further the effect of the induced flow, we focus on the mode $k=2.4$ to investigate its stability for different angles of propagation $\phi_{k}$. The inclination angel $S_{n}=0.4$ is considered in figure 9 . The mode for $\phi_{k}=90^{\circ}$, which propagates in the positive $y$-direction because $\omega_{i}$ is negative and therefore is a transverse mode, is the most stable mode and is of largest wave speed compared with the modes propagating in the other directions. This implies again that the induced flow stabilizes the modes in the same direction. The most unstable mode is for $\phi_{k}=0^{\circ}$ or $180^{\circ}$ (or the longitudinal mode) because the stabilizing effect due to the induced flow does not influence this 

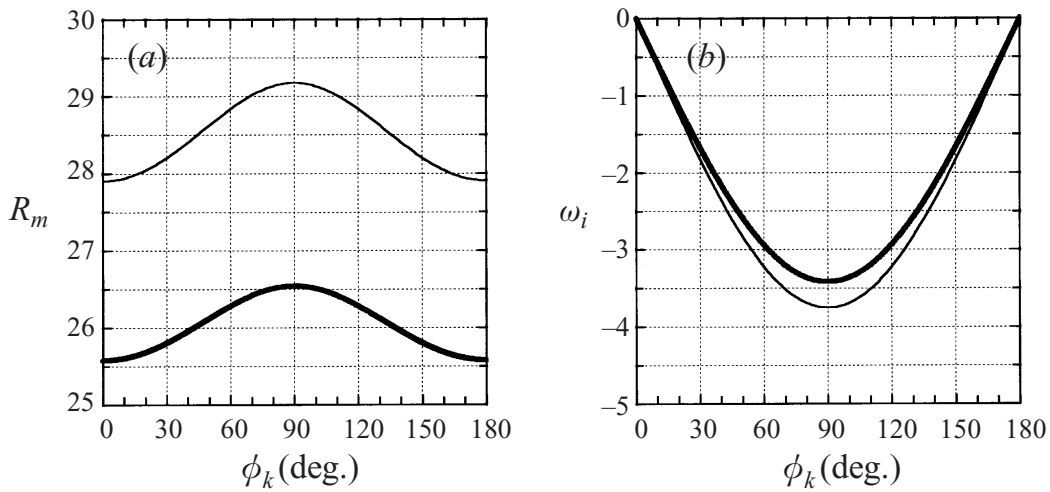

FIGURE 9. The variations of $R_{m}$ and $\omega_{i}$ with $\phi_{k}$ for the instability mode of $k=2.4$ and $S_{n}=0.4$.

Heavy curve: small- $\phi_{n}$ model. Fine curve: large- $\phi_{n}$ model.

mode. It is however noted that the precession also causes a rotation component in the $(x, y)$-plane, whose direction is aligned with the induced flow. Busse \& Kropp (1992) and Matthews \& Cox (1997) showed that when there is a rotation axis aligned with the direction of the shear flow, the most unstable buoyancy-driven instability mode is an oblique roll. However, for the present mush under inclined rotation in which an aqueous ammonium chloride solution is used, the effect of precession can be ignored if the precession speed is not high. It turns out that the present case approaches the limiting case of zero rotation of either Busse \& Kropp or Mathews \& Cox. Consequently, the most unstable mode is the longitudinal roll.

\section{Linear stability analysis II: precession-and-spin case}

When the cooling tank inclined with $\phi_{n}$ rotates with simultaneously precession and spin, the induced flow is a periodic function, varying with time with frequency equal to the spin angular velocity $\Omega$. We apply Fourier transformation with respect to both the $x$ - and $y$-directions to (4.1) and neglect the Coriolis effect because of the evidence given in the precession-only case, yielding

$$
\begin{gathered}
\left(\frac{\partial}{\partial t^{\prime}}-\frac{\partial}{\partial z}-\mathrm{i} \hat{U}_{b} k \sin \left(\Omega t^{\prime}\right)+k^{2}-\frac{\partial^{2}}{\partial z^{2}}\right) \hat{\theta}+\mathscr{F}\left(\frac{\partial}{\partial t^{\prime}}-\frac{\partial}{\partial z}\right) \hat{\chi}=-\theta_{b}^{\prime} \hat{w} \\
{\left[\chi_{b}\left(\frac{\partial}{\partial t^{\prime}}-\frac{\partial}{\partial z}\right)-\mathrm{i} \hat{U}_{b} k \sin \left(\Omega t^{\prime}\right)-\chi_{b}^{\prime}\right] \hat{\theta}+\left[\left(\theta_{b}-C\right)\left(\frac{\partial}{\partial t^{\prime}}-\frac{\partial}{\partial z}\right)-\theta_{b}^{\prime}\right] \hat{\chi}=-\theta_{b}^{\prime} \hat{w}} \\
\left(\frac{\partial^{2}}{\partial z^{2}}-k^{2}\right) \hat{w}=R_{m}\left\{\mathrm{i} S_{n} k \sin \left(\Omega t^{\prime}\right) \frac{\partial}{\partial z}+C_{n} k^{2}\right\} \hat{\theta}
\end{gathered}
$$

where $\hat{U}_{b}$ is basic flow induced by inclined precession and spin, defined as

$$
\hat{U}_{b}=S_{n} R_{m}\left(\mathscr{A} \theta_{\infty}+\theta_{b}\right),
$$

and $t^{\prime}$ is the time shift for the mode propagating in the direction of $\phi_{k}$, defined as

$$
t^{\prime} \equiv t+\frac{\phi_{k}}{\Omega} .
$$


When deriving (6.1), we find that the wavenumbers in the $x$ - and $y$-directions always appear together with the following relation:

$$
k_{x} \sin (\Omega t)+k_{y} \cos (\Omega t)=k \sin \left(\Omega t+\phi_{k}\right) .
$$

Thus, when $\Omega$ is larger than zero, through defining the time shift $t^{\prime}, \phi_{k}$ is absent from the governing equations, so the scale of computation can be greatly reduced. Equations (6.3) and (6.4) suggests that, for the present system spinning (with or without precession) with an inclined axis, an instability mode propagating in one direction has a phase which lags behind another mode propagating in a different direction by the so-called temporal-phase $\phi_{k} / \Omega$.

Equation (6.1) is a homogeneous differential equation, and can be solved by Floquet theory. The dependent variables are expanded into the following series:

$$
\left[\begin{array}{l}
\hat{\theta}(z, t) \\
\hat{w}(z, t) \\
\hat{\chi}(z, t)
\end{array}\right]=\sum_{|l| \leqslant L}\left[\begin{array}{l}
\theta_{l}(z) \\
w_{l}(z) \\
\chi_{l}(z)
\end{array}\right] \exp \left[(\omega+\mathrm{i} l \Omega) t^{\prime}\right],
$$

where $\omega=\omega_{r}+\mathrm{i} \omega_{i}$. The real part $\omega_{r}$ determines the stability of the basic state, namely the system is unstable when $\omega_{r}>0$, is stable when $\omega_{r}<0$, and is neutral when $\omega_{r}=0$. By substituting (6.5) into (6.1) and by applying the orthogonality of the Fouries series of (6.5), we obtain the following ordinary differential equations:

$$
\begin{gathered}
{\left[\mathrm{D}^{2}+\mathrm{D}-\left[k^{2}+\omega_{r}+\mathrm{i}\left(\omega_{i}+l \Omega\right)\right]\right] \theta_{l}+\frac{k U_{b}}{2}\left(\theta_{l-1}-\theta_{l+1}\right)} \\
+\mathscr{F}\left(D-\omega_{r}-\mathrm{i}\left(\omega_{i}+l \Omega\right)\right) \chi_{l}=\theta_{b}^{\prime} w_{l}, \\
{\left[\chi_{b} \mathrm{D}+\chi_{b}^{\prime}-\chi_{b}\left[\omega_{r}+\mathrm{i}\left(\omega_{i}+l \Omega\right)\right]\right] \theta_{l}+\frac{k U_{b}}{2}\left(\theta_{l-1}-\theta_{l+1}\right)} \\
+\left[\left(\theta_{b}-\mathscr{C}\right) \mathrm{D}+\theta_{b}^{\prime}-\left(\theta_{b}-\mathscr{C}\right)\left[\omega_{r}+\mathrm{i}\left(\omega_{i}+l \Omega\right)\right]\right] \chi_{l}=\theta_{b}^{\prime} w_{l}, \\
\left(\mathrm{D}^{2}-k^{2}\right) w_{l}=\left(\frac{k S_{n} R_{m}}{2}\right) \mathrm{D}\left(\theta_{l-1}-\theta_{l+1}\right)+C_{n} R_{m} k^{2} \theta_{l} .
\end{gathered}
$$

And the associated boundary conditions at $z=h_{b}$ are

$$
\theta_{l}=0, \quad \chi_{l}=0, \quad \mathrm{D} w_{l}=0,
$$

and at $z=0$ are

$$
\theta_{l}=0, \quad w_{l}=0 .
$$

In the above equations $|l| \leqslant L$. Equation (6.6) is a homogeneous equation of order of differentiation $10 L+5$. Equations (6.6), (6.7) and (6.8) constitute a complex eigenvalue problem, to be solved by the same shooting technique as employed in $\S 5$.

We consider again the $26 \%$ ammonium chloride solution. From several numerical tests we found that the number of terms of (6.5) required for a convergent solution decreases with increasing $\Omega$, as indicated by Murray et al. (1993). For $\Omega=500-50000$ (equivalent to $0.07-7$ r.p.m., $T_{a s}=10^{4}-10^{8}$ ), $L=5$ is large enough to make the iteration converge.

Note that, in the present coordinate system (the $x, y, z$ system in figure 1) spinning with the tank, gravity changes direction with frequency $\Omega$. Similarly, the induced flow (figure 3 ) in both the fluid and mushy layers is spinning with the frequency $\Omega$. This 

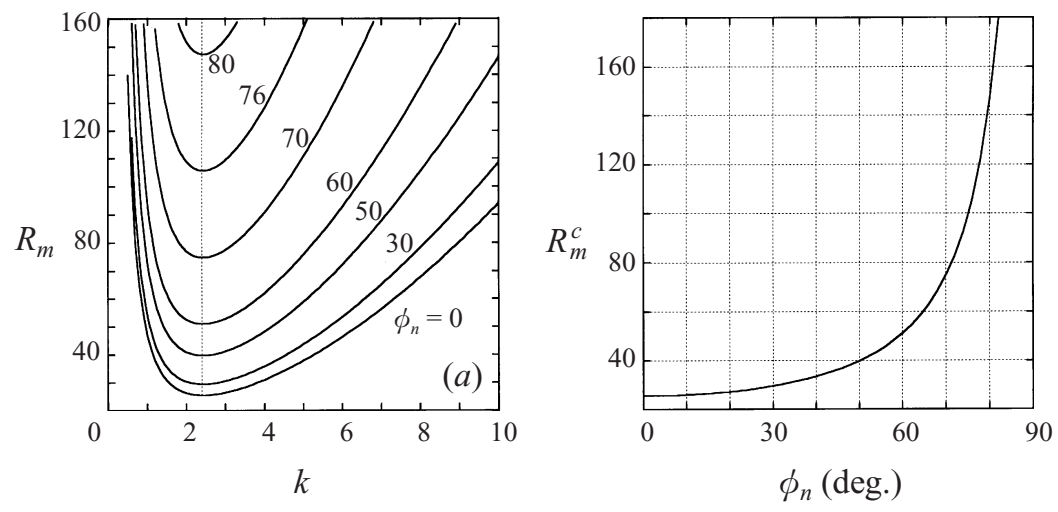

FIGURE 10. (a) The neutral curves of various $\phi_{n}$ and $(b)$ the variation of $R_{m}^{c}$ with $\phi_{n}$ for a system rotating with a spin angular velocity $\Omega=5000(\approx 0.7$ r.p.m.). All the instability modes are oscillatory, with a frequency equal to $\Omega$.

suggests that the instability mode is independent of the direction of propagation, namely the instability modes propagating in any direction have the same stability characteristics, e.g. the same $R_{m}, k, \omega_{i}$, etc.

Based on this we can explain the result shown in figure 10 as follows. Figure 10 illustrates the result of the case $\Omega=5000$ ( 0.7 r.p.m., showing the variation of $R_{m}$ with both $k$ and $\phi_{n}$. It is found that, when the system is spinning (with or without precession), the instability modes are synchronous with the spin because $\omega_{i}=0$, namely the instability mode varies its amplitude with time synchronously with the change of the directions of both gravity and the induced flow. The most critical mode for each different $\phi_{n}$ has the same critical wavenumber $k^{c}=2.4$. This critical mode may appear as a wave propagating in any direction, quite opposite to the precession-only case in which the most unstable wave propagates perpendicularly to the induced flow (i.e. the longitudinal mode). It is also noted that $R_{m}^{c}$ increases with $\phi_{n}$ (figure $10 b$ ), indicating that the system is more stable for a larger inclination angle. It is also found that the results for different $\Omega$ only show small differences, reflecting the fact that the spin effect on the system is applied through the Coriolis force, which has been neglected in the analysis since the induced flow is very small in the mush.

The relative importance of the induced flow and the buoyancy reduction along the thermal gradient can also be shown from figure 10. When the tank is inclined, the buoyancy reduces over the height of the tank by a factor of approximately $\cos \left(\phi_{n}\right)$. Applying this idea to figure 10, we find that $R_{m}^{c} \cos \left(\phi_{n}\right)$ is roughly constant at 25 . This implies that the primary stabilizing action comes from the buoyancy reduction. The induced flow has little influence on stabilizing the system. This is perhaps not surprising since the induced flow is very weak in the mush.

In the present system, a large number of terms (at least 10) are needed to get a convergent solution for the case of small spin rate, for example, $\Omega=O(1)$. However, fortunately, a spin rate of this order is equivalent to a very small r.p.m. value which is not realistic in either experiment or industry. As shown in $\S 3.2$, the induced flow is not valid for the case of low r.p.m. and we thus ignore this case in the present study. 


\section{Concluding remarks}

We have considered a system containing an aqueous ammonium chloride solution unidirectionally solidified from below, to which a rotation including spin and/or precession with an inclined axis is imposed. We first consider the two-layer system of a fluid layer overlying a mushy layer to examine the flow induced by the inclination. Based on this induced flow, which is obtained analytically, we implement a linear stability analysis for the flow in the mushy layer. The reason for making the simplification of considering only the mushy layer is twofold. First, the flow in the mush has been thought to be responsible for the formation of the freckles in metallic castings. Secondly, it is too lengthy to tackle the two-layer system, owing to the numerical difficulties. In brief, inclined rotation invariably stabilizes the flow in the mushy layer. This general conclusion is reached on the bases of the present results, which are summarized in the following categories: $(a)$ the induced flow, $(b)$ the stability in the mush and $(c)$ the limitations of the solution.

\section{(a) Induced flow}

(1) Neither the basic-state temperature nor concentration distributions of the system are influenced by the inclined rotation. The solute boundary layer is potentially unstable with respect to a small disturbance.

(2) The inclination induces a strong shear flow in the fluid layer, which is modified by the rotation. The maximum velocity is $O\left(10^{3}\right)$ to $O\left(14^{4}\right)$ larger than the induced flow in the mushy layer. The average velocity of the induced flow in the mush is of about the order as the speed of the freezing front. The induced flows in both layers move in the direction parallel to the flat fluid/mush interface (or the $x, y$-plane).

(3) The induced flow in the fluid layer is composed of three parts. First, there is a thermal-layer flow of thickness $O(1)$ in which the velocity decreases exponentially with height while its direction remains the same, leading gravity by $90^{\circ}$. Secondly, there is a solute-layer flow of thickness $O(\varepsilon)$ where both the velocity and the flow direction remain virtually the same in the whole fluid layer except in the shallow solute boundary layer above the fluid/mush interface, in which the velocity also decreases (although only a little) exponentially with height. Thirdly, there is an Ekman-layer flow of thickness $O\left(d_{E}\right)$ in which the velocity decreases exponentially with height dramatically and decays to zero shortly beyond the layer. The Ekman-layer flow changes its direction periodically with period $2 \pi / b$.

(4) The induced flow in the mush consists of two separated flows, drifting in the opposite directions, one at $180^{\circ}$ (or the $y$-direction, which is also the direction of gravity) and the other at $360^{\circ}$ with respect to the gravity component in the $(x, y)$-plane.

(5) For an aqueous solution, such as the ammonium chloride solution considered in the present study, $\varepsilon<d_{\varepsilon} \ll 1$. Both the solute boundary layer and the Ekman layer are confined to a shallow region above the interface while the thermal boundary layer represents a major part of the fluid layer.

(6) The velocity of the induced flow in the fluid layer increases when the inclination angle increases and decreases when the rotation speed in terms of $T_{e}$ increases. The velocity of the induced flow in the mush also increases when the inclination angle increases, but remains virtually unchanged on varying the speed of rotation, due to the resistance to the flow in the mush being high and the Coriolis force negligible.

(7) For the precession-only case, the induced flow is steady, pointing steadily in the same direction during the precession process. For the case including spin, the induced flow changes direction with frequency equal to the spin angular velocity $\Omega$, namely the induced flow scans all directions within a period $2 \pi / \Omega$. 
(b) Stability in the mush

(1) A larger inclination angle leads to a larger induced flow velocity and a greater reduction of the buoyancy in the $z$-direction, both resulting in a more stable state of the system. However, the primary stabilizing factor stems from the reduction of the buoyancy in $z$-direction because the induced flow is weak in the mush.

(2) For the precession-only case, the most-unstable mode is the longitudinal mode, which propagates in a direction perpendicular to the induced flow. This is because the Coriolis force is negligible due to the high resistance to flow in the mush.

(3) For the case including spin, the instability modes in all directions are of equal stability, because both the change in direction of the induced flow and the reduced buoyancy makes the stabilizing effect apply equally to the mode in each direction. The modes propagating in different directions differ from each other only by a phase difference, represented by the so-called temporal-phase $\phi_{k} / \Omega$.

(4) The rotation speed, due either to spin or precession, does not influence the stability of the mush since the Coriolis force is negligible. The inclination angle $\phi_{n}$ turns out to be the only factor influencing the stability.

\section{(c) Limits to the validity of the solution}

The limits to the validity of the present result may stem from many factors such as the validity of the induced flow solution derived in $\S 3$, in which several assumptions regarding the dimension of the system (or the cooling tank) have been made, the neglect of the coupling between the two layers, the neglect of the sidewall effects, and so on. We have discussed in $\S 3$ the validity of the induced flow solution; the corresponding assumptions are justified through a scale analysis of the equations, and the following conclusions are reached.

(1) For the precession-only case, the induced flow of $\S 3$ is valid only when the inclination angle is small and/or the precession speed is large. This condition is rather strict from the viewpoint of either laboratory or industry.

(2) For the case including spin, the induced flow of $\S 3$ is valid for a wide-range of physical dimensions, which satisfied most of the facilities used in either laboratory or industry.

We therefore conclude, from the viewpoint of laboratory and industrial application, that a scheme involving inclined spin can be a more efficient way to stabilize the unidirectional solidification system than inclined precession: in addition to the wide choice of dimensions, the system is of higher stability globally since the instability modes are stabilized in all directions due to the inclined spin.

A final comment is in order on the role played by inclined rotation in the stability of the present system. From the stability analysis in the mushy layer we found that the stabilizing effect due to gravity reduction is more significant than that due to induced flow (see figure $10 \mathrm{~b}$ ). We however note that, as the whole system including melt and mushy regions is considered, the induced flow could play an influential role in determining the stability in the melt. An interesting example can be seen in Feltham \& Worster (1999), who investigated the influence of a potential flow imposed parallel to the melt/mush interface on the stability of a system unidirectionally solidifying from above. They concluded that the imposed flow leads to a morphological instability mode on the interface, above which a series of small convection cells form. In fact, we have obtained similar results (not published) for a system unidirectionally solidifying from below. In the present system, there is a strong shear flow forming above the interface when the tank is under inclined rotation, but such an instability mode has never been found either by the present theoretical analysis or in the experiment of 
Sample \& Hellawell (1984). We conjecture that, since the induced velocity decreases as the rotation speed increases, the rotation (either precession or spin) has indeed helped inhibit the morphological instability. To understand more thoroughly the influence of induced flow on the present system, further research on the effect of inclined precession/spin on the whole system is required.

The financial support for this work and the post-doctoral fellowship for CAC from National Science Council through Grant No. NSC 88-2212-E-002-018 is gratefully acknowledged.

\section{REFERENCES}

Amberg, G. \& Homsy, G. M. 1993 Nonlinear analysis of buoyant convection in binary solidification with application to channel formation. J. Fluid Mech. 252, 79-98.

Anderson, D. M. \& Worster, M. G. 1995 Weakly nonlinear analysis of convection in a mushy layer during the solidification of binary alloys. J. Fluid. Mech. 302, 307-331.

Beavers, G. S. \& Joseph, D. D. 1967 Boundary conditions at a naturally permeable wall. J. Fluid Mech. 30, 199-207.

Busse, F. H. \& Kropp, M. 1992 Buoyancy driven instabilities in rotating layers with parallel axix of rotation. Z. Angew. Math. Mech. 43, 28-35.

CHEN, C. F. \& CHEN, F. 1991 Experimental study of directional solidification of aqueous ammonium chloride solution. J. Fluid Mech. 227, 567-586.

Chen, F., Lu, J. W. \& YANG, T. L. 1994 Convective instability in ammonium chloride solution directionally solidified from below. J. Fluid Mech. 276, 163-187.

Chen, F., YAng, T. L. \& LU, J. W. 1993 Influence of convection on solidification of binary solutions cooling from below. J. Appl. Phys. 74, 7531-7541.

Chung, C. A. \& Chen, F. 2000 Onset of plume convection in mushy layer. J. Fluid Mech. 408, 53-82.

DAvis, S. H. 1990 Hydrodynamic interactions in directional solidification. J. Fluid Mech. 212, 241-262.

Emms, P. W. \& Fowler, A. C. 1994 Compositional convection in the solidification of binary alloys J. Fluid Mech. 262, 111-139.

Feltham, D. L. \& Worster, M. G. 1999 Flow-induced morphological instability of a mushy layer. J. Fluid Mech. 391, 337-357.

Forth, S. A. \& Wheeler, A. A. 1992 Coupled convective and morphological instability in a simple model of the solidification of a binary alloy, including a shear flow. J. Fluid Mech. 236, 61-94.

Huppert, H. E. 1990 The fluid mechanics of solidification. J. Fluid Mech. 212, 209-240.

Huppert, H. E. \& Hallworth, M. A. 1993 Solidification of $\mathrm{NH}_{4} \mathrm{Cl}$ and $\mathrm{NH}_{4} \mathrm{Br}$ from aqueous solutions contaminated by $\mathrm{CuSO}_{4}$ : the extinction of chimneys. J. Cryst. Growth 130, 495-506.

Ingersoll, A. P. 1966 Convective instabilities in plane Couette flow. Phys. Fluids 9, 682-689.

Keller, H. B. 1976 Numerical Solutions of Two Point boundary Value problems. SIAM.

LANGLOIS, W. E. 1985 Buoyancy-driven flows in crystal growth melts. Ann. Rev. Fluid Mech. 17, 191-215.

Linden, P. F. 1974 Salt fingers in a steady shear flow. Geophys. Fluid Dyn. 6, 1-27.

Lu, J. W. \& Chen, F. 1997 Rotation effects on the convection of binary alloys unidirectional solidified from below. Intl J. Heat Mass Transfer 40, 237-246.

Matthews, P. \& Cox, S. 1997 Linear stability of rotating convection in an imposed shear flow. $J$. Fluid Mech. 350, 271-293.

Mullins, W. W. \& Sekerka, R. F. 1964 Stability of a planar interface during solidification of a binary alloy. J. Appl. Phys. 35, 444-451.

Murray, B. T., Coriell, S. R., McFadden, G. B., Wheeler, A. A. \& Saunders, B. V. 1993 Gravitational modulation of thermosolutal convection during directional solidification. $J$. Cryst. Growth 129, 70-80.

NeILson, D. G. \& InCropera, F. P. 1992 Effect of rotation on fluid motion and channel formation during unidirectional solidification of a binary alloy. Intl J. Heat Mass Transfer 36, 489-505. 
Powell, M. J. 1970 Numerical Methods for Nonlinear Algebraic Equations. Gordon \& Breach.

Sample, A. K. \& Hellawell, A. 1984 The mechanism of formation and prevention of channel segregation during alloy solidification. Metall. Trans. A 15, 2163-2173.

TAIT, S. \& JAUPART, C. 1992 Compositional convection in a reactive crystalline mush and melt differentiation. J. Geophys. Res. 97 (B5), 6735-6759.

Worster, M. G. 1986 Solidification of an alloy from a cooled boundary. J. Fluid Mech. 167, 481-501.

Worster, M. G. 1992 Instabilities of the liquid and mushy regions during solidification of alloys. $J$. Fluid Mech. 237, 649-669.

Worster, M. G. \& Kerr, R. C. 1994 The transient behavior of alloys solidified from below prior to the formation of chimneys. J. Fluid Mech. 269, 23-44. 